\title{
ON STOCHASTIC SET FUNCTIONS. I
}

\author{
András Prékopa (Budapest) \\ (Presented by A. Rényi) \\ Institution for Applied Mathematics of the Hungarian Academy of Sciences
}

Received: May 18, 1956

\section{Introduction}

The theory of stochastic processes, as it has been founded by Kolmogorov in 1931, originally dealt with the mathematical treatment of random time processes. In later years, however, there arose many practical problems which are not random time processes but the method of solution of which was similar to those used in the theory of stochastic processes. Such problems occur e.g. when investigating the spatial distribution of stars or colloid particles, when counting bloodcells, when investigating the quantity of rain or crop in a given area etc.

There is, however, an essential difference between the mathematical models of the above mentioned problems and that of those described by random time processes. In a time process we are generally interested only in the state of the system at the moment $t$, which is characterised by a random variable $\xi_{t}$, when considering the random distribution of points in the plane, however, the state of the point system will be described most perfectly by random variables attached to sets of the plane and not by such that are attached to the points of the plane. If $A$ is a set of the plane, then there corresponds to $A$ a random variable $\xi(A)$ which gives the number of points in the set $A$. Thus $\xi(A)$ is a random set function.

The subject of this paper is the investigation of some problems concerning such random set functions. I introduce the notions of stochastic additive and stochastic completely additive set functions and examinate problems some of which are generalizations of problems arising in the theory of real-valued set functions or similar to them and some of which are of probabilistic nature.

Definition 1. Let $\mathcal{R}$ be a ring consisting of some subsets of a space $H$. Suppose that to every element $A$ of $\mathcal{R}$ there corresponds a random variable $\xi(A)=\xi(\omega, A)$ such 
that if $A_{1}, A_{2}, \ldots, A_{r}$ are disjoint sets belonging to the ring $\mathcal{R}$, then the random variables $\xi\left(A_{1}\right), \xi\left(A_{2}\right), \ldots, \xi\left(A_{r}\right)$ are independent and

$$
\xi(A)=\sum_{k=1}^{r} \xi\left(A_{k}\right) ;
$$

in this case $\xi(A)$ will be called a stochastic additive set function.

Definition 2. A stochastic additive set function $\xi(A)$ defined on a ring $\mathcal{R}$ will be called a stochastic completely additive set function if for every sequence $A_{1}, A_{2}, \ldots$ of disjoint sets of $\mathcal{R}$, for which $A=\sum_{k=1}^{\infty} A_{k} \in \mathcal{R}$, the equality

$$
\xi(A)=\sum_{k=1}^{\infty} \xi\left(A_{k}\right)
$$

is satisfied.

For the sake of brevity, the word "stochastic" will be omitted and if an ordinary realvalued additive (or completely additive) set function will be considered, this will be mentioned explicitly.

The idea of random set functions has appeared first in a paper of S. BochneR [2]. The notion introduced by him is similar to that introduced by Definition 1 but in [2] it is not required that the random variables belonging to disjoint sets should be independent. It is possible to make further generalizations, for instance, taking instead of the ring $\mathcal{R}$ a Boolean algebra, or instead of a single random variable $\xi(A)$ a random vector $\vec{\xi}(A)=$ $\left(\xi_{1}(A), \xi_{2}(A), \ldots, \xi_{n}(A)\right)$ etc. Some special random set functions have been considered by H. Cramér [3] , E. Marczewski [9], C. RYll-Nardzewski [12], further by A BlanCLAPIERRE and R. ForTet [1].

From the point of view of practical applications it is important that if e.g. $H$ is some finite-dimensional Euclidean space, there should correspond a random variable to any sphere, domain, and eventually even to more complicated sets. As it will be shown in $\S 1$ of Chapter III, by the construction of Kolmogorov $([6] \S 4)$ we can construct stochastic additive set functions which are defined on intervals or on finite sums of intervals but the general problem cannot be solved by this method. In order to get further we have to deal with the problem of the extension of stochastic set functions.

The main purpose of this paper is the extension of a stochastic completely additive set function defined on a ring $\mathcal{R}$ and satisfying certain conditions, to the smallest $\sigma$-ring $\mathcal{S}(\mathcal{R})$ which contains $\mathcal{R}$.

The extension of some special stochastic set functions defined on sets of Euclidean spaces has been considered by H. Cramér [3], E Marczewski [9] and C. RYll-NardZEWSKI [12]. The theorems of the present paper contain as special cases the relevant 
theorems of [9] and [12]. In [3] a theorem is proved concerning the extension of a random set function generated by the differences of an ordinary one-dimensional stochastic process.

The problem of extension of stochastic set functions is a generalization of the convergence problem of the series of independent random variables. Namely, if $\xi_{1}, \xi_{2}, \ldots$ is a sequence of independent random variables and $\mathcal{R}$ is the ring of the finite sets of the natural numbers, then the set function

$$
\xi(A)=\sum_{k \in A} \xi_{k} \quad(A \in \mathcal{R})
$$

can be extended to the $\sigma$-ring $\mathcal{S}(\mathcal{R})$ if and only if the series $\sum_{k=1}^{\infty} \xi_{k}$ converges with probability 1 regardless of the order of summation ([4], p. 118, Corollary 1).

There is also another way to construct the theory of random set functions. The theory of stochastic processes can be built up by considering the space of functions of a real variable and defining a measure in this space. As it has been proved by E. HopF [5] the same procedure can be carried out also in the space of additive set functions defined on a ring $\mathcal{R}$. The measure thus defined is, however, only finitely additive. From several points of view, however, it is necessary that the probability should be completely additive. Thus the extension problem arises in another connection also here. But in this case the fulfilment of the conditions ensuring the possibility of extension is not a simple problem even in particular cases.

The notion of a stochastic additive set function is in some sense a generalization of the notion of a process with independent increments. Namely, we are often interested in the differences $\xi_{t_{2}}-\xi_{t_{1}}$ only; these and their finite sums can, however, be regarded as a stochastic additive set function.

I wish to express my thanks to Professors A. RÉnYi, B. SzŐKefalvi-NaGy and Á. CsÁszÁr for their valuable remarks.

\section{Definitions and notations}

Let $H$ be an arbitrary set and $\mathcal{R}$ a class of sets of some subsets of $H$. The class $\mathcal{R}$ of sets will be called a ring if $A+B \in \mathcal{R}, A-B \in \mathcal{R}$, provided that $A \in \mathcal{R}, B \in \mathcal{R}$. If $H \in \mathcal{R}$, then $\mathcal{R}$ is called an algebra. If for an arbitrary sequence $A_{1}, A_{2}, \ldots$ of sets of the ring (algebra) $\mathcal{R}$ we have $\sum_{k=1}^{\infty} A_{k} \in \mathcal{R}$, then $\mathcal{R}$ will be called a $\sigma$-ring ( $\sigma$-algebra). If $\mathcal{R}$ is a ring ( $\sigma$-ring) and $A \in \mathcal{R}$, then $A \mathcal{R}$ denotes the algebra ( $\sigma$-algebra), the elements of which are those subsets of $A$ which belong to the $\operatorname{ring} \mathcal{R}$. $\mathcal{S}(\mathcal{R})$ denotes the smallest $\sigma$-ring containing the ring $\mathcal{R}$.

A real-valued, non-negative set function $m(A)$ defined on the elements of a ring $\mathcal{R}$ will be called a measure, if for every sequence $A_{1}, A_{2}, \ldots$ of disjoint sets of $\mathcal{R}$ for which 
$A=\sum_{k=1}^{\infty} A_{k} \in \mathcal{R}$ we have

$$
m(A)=\sum_{k=1}^{\infty} m\left(A_{k}\right) .
$$

If $m(A)$ may have also negative values but the relation (2) holds without any restriction, then $m(A)$ will be called a completely additive set function.

The $n$-dimensional Euclidean space is denoted by $R_{n}$. If $A$ is a Lebesgue measurable set of the space $R_{n}$, then $|A|$ denotes its $n$-dimensional Lebesgue measure.

The random variables which we consider in this paper are all supposed to be defined on the same space of elementary events $\Omega$. The elements of $\Omega$ are denoted by $\omega$. We suppose that there is a $\sigma$-algebra $\mathcal{T}$ consisting of some subsets of the space $\Omega$ and on the elements of $\mathcal{T}$ a probability measure $\mathbf{P}$ is defined for which $\mathbf{P}(\Omega)=1$. The measurable functions defined on the space $\Omega$, which are finite-valued almost everywhere, are called random variables. If $\xi$ and $\eta$ are two random variables, then the relation $\xi=\eta$ means that

$$
\mathbf{P}(\xi=\eta)=1 \text {. }
$$

Inequalities between random variables have similar meaning. If $\xi_{1}, \xi_{2}, \ldots$ is a sequence of random variables, then the relation $\xi_{k} \rightarrow \xi\left(\right.$ or $\lim _{k \rightarrow \infty} \xi_{k}=\xi$ ) denotes that

$$
\mathbf{P}\left(\lim _{k \rightarrow \infty} \xi_{k}=\xi\right)=1 .
$$

The relation $\xi_{k} \Rightarrow \xi\left(\right.$ or $\lim _{k \rightarrow \infty}$ st $\xi_{k}=\xi$ ) denotes that for every positive $\varepsilon$

$$
\lim _{k \rightarrow \infty} \mathbf{P}\left(\left|\xi_{k}-\xi\right|>\varepsilon\right)=0 .
$$

If $f_{k}(t)$ and $f(t)$ are the characteristic functions of $\xi_{k}$ and $\xi$, respectively, and $f_{k}(t) \rightarrow f(t)$ for every value of $t$ (or what is the same, the convergence is uniform in every finite $t$ interval), then this will be expressed by

$$
f_{k}(t) \Rightarrow f(t)
$$

(4) follows from (3), and if $\xi=0$, then (3) follows from (4).

If $\xi$ is a random variable and for the real number $Q(\lambda)$ the relations

$$
\mathbf{P}(\xi \leq Q(\lambda)) \geq \lambda, \quad \mathbf{P}(\xi \geq Q(\lambda)) \geq 1-\lambda,
$$

(where $0<\lambda<1$ ) are satisfied, then the number $Q(\lambda)$ will be called a $\lambda$-quantile of the random variable $\xi$. 


\section{AUXILIARY THEOREMS}

\section{$\S 1$. Set functions which are subadditive or of bounded vari- ation}

Definition. Let $\mathcal{A}(A, B, \ldots)$ be a class of sets. A real-valued set function $\alpha(A)$ defined on the elements of $\mathcal{A}$ will be called of bounded variation if there is a number $K$ such that for every system of sets $A_{1}, A_{2}, \ldots, A_{r}$, consisting of disjoint sets belonging to $\mathcal{A}$, the relation

$$
\sum_{k=1}^{r}\left|\alpha\left(A_{k}\right)\right| \leq K
$$

holds.

The smallest number $K$ for which the preceding inequality holds, is called the variation of $\alpha$. If only sets $A_{k} \subseteq A(k=1,2, \ldots, r)$ are admitted, then the number

$$
\sup _{\left\{A_{k}\right\}} \sum_{k=1}^{r}\left|\alpha\left(A_{k}\right)\right|
$$

will be called the variation of the set function $\alpha$ in $A$. We denote this quantity by $\operatorname{Var}_{\alpha}(A)$.

Definition. Let $\mathcal{A}(A, B, \ldots)$ be a class of sets and $\alpha(A)$ a real-valued set function defined on the elements of $\mathcal{A}$. We call the set function $\alpha$ subadditive (superadditive) if for every system $A_{1}, A_{2}, \ldots, A_{r}$, consisting of disjoint sets belonging to $\mathcal{A}$, for which $A=\sum_{k=1}^{r} A_{k} \in \mathcal{A}$, the relation

$$
\alpha(A) \leq \sum_{k=1}^{r} \alpha\left(A_{k}\right) \quad\left(\alpha(A) \geq \sum_{k=1}^{r} \alpha\left(A_{k}\right)\right)
$$

holds.

If a set function $\alpha$ is both subadditive and superadditive, we say that $\alpha$ is an additive set function. If in the above inequality $r$ may be also infinite, then $\alpha$ will be called completely subadditive (completely superadditive).

If $\alpha$ is a set function of bounded variation, then the set function $\operatorname{Var}_{\alpha}(A)$ is obviously completely superadditive. We shall often use the following theorems which are proved in [11].

THEOREM 1.1. Let $\mathcal{R}$ be a ring and $\alpha$ a real-valued, non-negative set function defined on $\mathcal{R}$ for which the following conditions are satisfied: 
a) $\alpha(A) \leq K$ where $K$ is a constant;

b) $\alpha(A)$ is subadditive;

c) if $A_{1}, A_{2}, \ldots$ is a sequence of disjoint sets of $\mathcal{R}$, then

$$
\sum_{k=1}^{\infty} \alpha\left(A_{k}\right)<\infty
$$

Under these conditions $\alpha(A)$ is a bounded variation.

THEOREM 1.2. Let $\mathcal{R}$ be a ring and $\alpha(A)$ a completely subadditive set function of bounded variation. Then the set function $\operatorname{Var}_{\alpha}(A)(A \in \mathcal{R})$ is a bounded measure on $\mathcal{R}$.

\section{$\S 2$. Some inequalities}

In this $\S$ we derive some inequalities which we shall use later. In advance we mention the following elementary inequalities:

$$
\begin{array}{lll}
1-\frac{\sin x}{x} \geq \frac{x^{2}}{8} & \text { if } & |x| \leq 1, \\
1-\frac{\sin x}{x} \geq 1-\frac{\sin \varepsilon}{\varepsilon} & \text { if } & 0<\varepsilon \leq 1 \text { and }|x| \geq \varepsilon, \\
1-\frac{\sin x}{x}>\frac{1}{10} & \text { if } & |x| \geq 1 .
\end{array}
$$

Let $\xi$ be a random variable, $F(x)$ and $f(t)$ denote its distribution function and characteristic function, respectively. If $0<\varepsilon \leq 1$, then using the preceding inequalities we obtain

$$
\begin{aligned}
& \frac{1}{2} \int_{-1}^{1}|1-f(t)| \mathrm{d} t \geq \frac{1}{2}\left|\int_{-1}^{1}(1-f(t)) \mathrm{d} t\right|=\frac{1}{2}\left|\int_{-1}^{1} \int_{-\infty}^{\infty}\left(1-e^{i t x}\right) \mathrm{d} F(x) \mathrm{d} t\right| \\
& \quad=\int_{-\infty}^{\infty}\left(1-\frac{\sin x}{x}\right) \mathrm{d} F(x) \geq \frac{1}{8} \int_{|x| \leq \varepsilon} x^{2} \mathrm{~d} F(x)+\left(1-\frac{\sin \varepsilon}{\varepsilon}\right) \mathbf{P}(|\xi|>\varepsilon) .
\end{aligned}
$$

Hence

$$
\frac{1}{2} \int_{-1}^{1}|1-f(t)| \mathrm{d} t \geq \min \left(\frac{1}{8}, 1-\frac{\sin \varepsilon}{\varepsilon}\right)\left[\int_{|x| \leq \varepsilon} x^{2} \mathrm{~d} F(x)+\mathbf{P}(|\xi|>\varepsilon)\right] .
$$

Let $\delta$ be an arbitrary positive number. Then analogously we obtain

$$
\begin{aligned}
\frac{1}{2 \delta} \int_{-\delta}^{\delta}|1-f(t)| \mathrm{d} t & \geq \int_{-\infty}^{\infty}\left(1-\frac{\sin \delta x}{\delta x}\right) \mathrm{d} F(x) \\
& \geq \int_{|x|>\frac{1}{\delta}}\left(1-\frac{\sin \delta x}{\delta x}\right) \mathrm{d} F(x) \geq \frac{1}{10} \mathbf{P}\left(|\xi|>\frac{1}{\delta}\right) .
\end{aligned}
$$


We shall often use the following well-known inequality: if $f(t)$ is a characteristic function, then

$$
1-\mathfrak{R} f(2 t) \leq 4(1-\mathfrak{R} f(t))^{1}
$$

(see e.g. [5], p. 61).

The following theorem is a straightforward generalization of a lemma in [4], p. 113.

Theorem 1.3. Let $\xi$ be a random variable and denote $Q(\lambda)$ a $\lambda$-quantile of $\xi$. Let $z$ be an arbitrary real number and $s(x)$ a non-negative function such that if $x \geq z$, then $s(x)$ is non-decreasing and if $x \leq z$, it is non-increasing. We suppose further that $M[s(\xi-\eta)]$ exists where $\eta$ is a random variable having the same distribution as $\xi$ and independent of $\xi$. Under these conditions $M[s(\xi-Q(\lambda))]$ also exists and the following inequality holds:

$$
M[s(\xi-\eta)] \geq \min (\lambda, 1-\lambda) M[s(\xi-Q(\lambda))] .
$$

Proof. Let $\xi_{1}=\xi-Q(\lambda), \eta_{1}=\eta-Q(\lambda)$. Then the value 0 is a $\lambda$-quantile of $\xi_{1}$. Denote $F_{1}(x)$ the common distribution function of $\xi_{1}$ and $\eta_{1}$. Then we have

$$
\begin{aligned}
& M[s(\xi-\eta)]=M\left[s\left(\xi_{1}-\eta_{1}\right)\right]=\int_{-\infty}^{\infty} \int_{-\infty}^{\infty} s(x-y) \mathrm{d} F_{1}(x) \mathrm{d} F_{1}(y) \\
& \quad \geq \int_{x \geq z} \int_{y \leq 0} s(x-y) \mathrm{d} F_{1}(x) \mathrm{d} F_{1}(y)+\int_{x<z} \int_{y \geq 0} s(x-y) \mathrm{d} F_{1}(x) \mathrm{d} F_{1}(y) \\
& \quad \geq \int_{y \leq 0} \mathrm{~d} F_{1}(y) \int_{x \geq z} s(x) \mathrm{d} F_{1}(x)+\int_{y \geq 0} \mathrm{~d} F_{1}(y) \int_{x<z} s(x) \mathrm{d} F_{1}(x) \\
& \quad \geq \min (\lambda, 1-\lambda) \int_{-\infty}^{\infty} s(x) \mathrm{d} F_{1}(x)=\min (\lambda, 1-\lambda) M[s(\xi-Q(\lambda))] .
\end{aligned}
$$

COROllary. If $s(x)$ is the function defined by

$$
s(x)=\left\{\begin{array}{ll}
0 & \text { if }|x| \leq \varepsilon \\
1 & \text { if }|x|>\varepsilon
\end{array} \quad(\varepsilon>0),\right.
$$

then the inequality (1.4) gives

$$
\mathbf{P}(|\xi-\eta|>\varepsilon) \geq \min (\lambda, 1-\lambda) \mathbf{P}(|\xi-Q(\lambda)|>\varepsilon) .
$$

From the inequalities (1.2) and (1.5) it follows that

$$
\begin{aligned}
\frac{1}{2 \delta} \int_{-\delta}^{\delta}\left(1-|f(t)|^{2}\right) \mathrm{d} t & >\frac{1}{10} \mathbf{P}\left(|\xi-\eta|>\frac{1}{\delta}\right) \\
& \geq \frac{\min (\lambda, 1-\lambda)}{10} \mathbf{P}\left(|\xi-Q(\lambda)|>\frac{1}{\delta}\right) .
\end{aligned}
$$

\footnotetext{
${ }^{1} \mathfrak{R}_{z}$ denotes the real part of the complex number $z$.
} 


\section{$\S 3$. Boundedness of sets of quantiles}

Let $Z$ be an arbitrary set and $\xi_{z}(z \in Z)$ a family of random variables. Denote by $Q(\lambda, z)$ a $\lambda$-quantile of the variable $\xi_{z}$. If for every $z$ we choose a $Q(\lambda, z)$, then we obtain a set: $\{Q(\lambda, z), z \in Z\}$ (this set is uniquely determined or not according to whether the quantiles $Q(\lambda, z)$ for every $z$ being uniquely determined or not).

Let us define the following quantities:

$$
\mu_{1}=\lim _{\varepsilon \rightarrow \infty} \sup _{z \in Z} \mathbf{P}\left(\xi_{z}<-\varepsilon\right), \quad \mu_{2}=\lim _{\varepsilon \rightarrow \infty} \sup _{z \in Z} \mathbf{P}\left(\xi_{z}>\varepsilon\right) .
$$

The connection between the variables $\xi_{z}$ and the quantities $\mu_{1}, \mu_{2}$ is shown by the following

THEOREM 1.4. If the quantiles $Q(\lambda, z)$ can be chosen in such a way that the set $\{Q(\lambda, z)$, $z \in Z\}$ is bounded from below (from above), then we have

$$
\mu_{1} \leq \lambda \quad\left(\mu_{2} \leq 1-\lambda\right) .
$$

If the quantiles $Q(\lambda, z)$ can be chosen in such a way that the set $\{Q(\lambda, z), z \in Z\}$ is not bounded from below (from above), then

$$
\mu_{1} \geq \lambda \quad\left(\mu_{2} \geq 1-\lambda\right) .
$$

Proof. Let us first prove the first half of the inequalities (1.7) and (1.8). The proof of the statements in the brackets proceeds in a similar way. Suppose that there is a number $K(\lambda)$ for which the inequality $Q(\lambda, z) \geq K(\lambda)(z \in Z)$ holds with a convenient choice of the quantiles $Q(\lambda, z)$, and suppose that $\mu_{1}>\lambda$. Then, for every $\varepsilon$, the relation

$$
\sup _{z \in Z} \mathbf{P}\left(\xi_{z}<-\varepsilon\right)>\lambda
$$

will be fulfilled. Let $\varepsilon_{0}$ be a number such that $-\varepsilon_{0} \leq K(\lambda)$ and let us choose a $z_{0}$ for which

$$
\mathbf{P}\left(\xi_{z_{0}}<-\varepsilon_{0}\right)>\lambda .
$$

Hence it follows that for every $\lambda$-quantile $Q\left(\lambda, z_{0}\right)$ of the variable $\xi_{z_{0}}$ the relation

$$
Q\left(\lambda, z_{0}\right)<-\varepsilon_{0} \leq K(\lambda)
$$

holds which is a contradiction. Consequently, the first half of (1.7) is true.

Now suppose that the set $\{Q(\lambda, z), z \in Z\}$ is not bounded from below with a convenient choice of the quantiles $Q(\lambda, z)$. Then, for every $\varepsilon$, we can find a $z_{\varepsilon}$ such that

$$
\mathbf{P}\left(\xi_{z_{\varepsilon}}<-\varepsilon\right) \geq \lambda
$$


It follows that for every $\varepsilon$ the relation

$$
\sup _{z \in Z} \mathbf{P}\left(\xi_{z}<-\varepsilon\right) \geq \lambda
$$

holds, hence

$$
\mu_{1}=\lim _{\varepsilon \rightarrow \infty} \sup _{z \in Z} \mathbf{P}\left(\xi_{z}<-\varepsilon\right) \geq \lambda .
$$

Thus we have proved also the first half of (1.8).

COROLlary. It follows from the inequalities (1.7) and (1.8) that, for every fixed value of $\lambda(0<\lambda<1)$, the $\operatorname{sets}^{2}\{Q(\lambda, z), z \in Z\}$ will be bounded from below (from above) if and only if $\mu_{1}=0\left(\mu_{2}=0\right)$. Let us define the quantity

$$
\mu_{3}=\lim _{\varepsilon \rightarrow \infty} \sup _{z \in Z} \mathbf{P}\left(\left|\xi_{z}\right|>\varepsilon\right)
$$

Clearly we have

$$
\mu_{1} \leq \mu_{3}, \quad \mu_{2} \leq \mu_{3}, \quad \mu_{3} \leq \mu_{1}+\mu_{2} .
$$

Hence it follows that for every fixed value of $\lambda(0<\lambda<1)$ the sets $\{Q(\lambda, z), z \in Z\}$ will be bounded if and only if $\mu_{3}=0$.

Similarly, from the inequalities (1.7) and (1.8) we obtain also the following statements:

For every fixed value of $\lambda$ the sets $\{Q(\lambda, z), z \in Z\}(0<\lambda<1)$ are not bounded from below (from above) if and only if $\mu_{1}=1\left(\mu_{2}=1\right)$.

For every fixed value of $\lambda$ the sets $\{Q(\lambda, z), z \in Z\}(0<\lambda<1)$ are bounded if and only if $\mu_{3}=1$.

\section{$\S 4$. Compactness of sets of distribution functions}

Denote by $\mathcal{F}$ the set of one-dimensional distribution functions. P. LÉvY has introduced the notion of the distance of two distribution functions. The distance of the distribution functions $F_{1}(x)$ and $F_{2}(x)$ is defined as the lower bound of those values $h$ for which the following inequality holds:

$$
F_{1}(x-h)-h \leq F_{2}(x) \leq F_{1}(x+h)+h \quad(-\infty<x<+\infty) .
$$

Let us denote this number by $L\left(F_{1}, F_{2}\right)$. Clearly $L\left(F_{1}, F_{2}\right) \leq 1$. It is known that this distance satisfies the axioms of metric spaces, i.e.

\footnotetext{
${ }^{2}$ If we speak about the boundedness (unboundedness) of the sets $\{Q(\lambda, z), z \in Z\}$ for every fixed $\lambda$ $(0<\lambda<1)$, the special choice of the quantiles $Q(\lambda, z)$ does not matter as in this case the boundedness (unboundedness) of the sets $\{Q(\lambda, z), z \in Z\}$ with a special choice of the quantiles $Q(\lambda, z)$ implies the boundedness of the sets $\{Q(\lambda, z), z \in Z\}$ with every choice of the quantiles. In this case we may interpret $\{Q(\lambda, z), z \in Z\}$ as the set of all the $\lambda$-quantiles of all the variables $\xi_{z}$, too.
} 
a) $L\left(F_{1}, F_{2}\right)=0$ if and only if $F_{1} \equiv F_{2}$;

b) $L\left(F_{1}, F_{2}\right)=L\left(F_{2}, F_{1}\right)$;

c) $L\left(F_{1}, F_{3}\right) \leq L\left(F_{1}, F_{2}\right)+L\left(F_{2}, F_{3}\right)$.

It is also known that the space $\mathcal{F}$ is complete with respect to the distance of LÉVI ([5], p. 42, Theorem 2). Consequently, $\mathcal{F}$ is a bounded and complete but non-compact metric space. In what follows we shall deal with the question, under what condition a subset $\mathcal{F}^{\prime}$ of the space $\mathcal{F}$ will be compact.

TheOREm 1.5. A subset $\mathcal{F}^{\prime}=\{F(x, z), z \in Z\}$ of the space $\mathcal{F}$ is compact if and only if the set $\{Q(\lambda, z), z \in Z\}(0<\lambda<1)$ is bounded for every $\lambda$.

Proof. According to the Corollary to Theorem 1.4 the sets $\{Q(\lambda, z), z \in Z\}$ will be bounded for every fixed value of $\lambda$ if and only if

$$
\lim _{\varepsilon \rightarrow \infty} \sup _{z \in Z}[F(-\varepsilon, z)+1-F(\varepsilon+0, z)]=0 .
$$

This is equivalent with

$$
\sup _{z \in Z} F(-\varepsilon, z) \rightarrow 0, \quad \inf _{z \in Z} F(\varepsilon, z) \rightarrow 1 \quad \text { if } \quad \varepsilon \rightarrow \infty .
$$

Consequently, if $F\left(x, z_{1}\right), F\left(x, z_{2}\right), \ldots$ is a sequence of distribution functions belonging to $\mathcal{F}^{\prime}$ and a subsequence $F\left(x, z_{n_{k}}\right)$ of this sequence converges ${ }^{3}$ to a non-decreasing function $F(x)$, which is continuous on the left, at every point of continuity of the latter, then $F(-\infty)=0$ and $F(+\infty)=1$.

TheOREM 1.6. A set $\mathcal{F}^{\prime}=\{F(x, z), z \in Z\}$ of the space $\mathcal{F}$ is compact if and only if $\mu_{3}=0$.

Proof. The theorem is a straightforward consequence of Theorem 1.5 and of the Corollary of Theorem 1.4.

Theorem 1.7. A set $\mathcal{F}^{\prime}=\{F(x, z), z \in Z\}$ of the space $\mathcal{F}$ is compact if and only if the characteristic functions $f(t, z)(z \in Z)$ are equally continuous at the point $t=0$, i.e. to every positive $\varepsilon$ there belongs a $\delta>0$ such that

$$
|1-f(t, z)|<\varepsilon \quad \text { if } \quad|t|<\delta .
$$

Proof. Suppose the set $\mathcal{F}^{\prime}$ to be compact. Hence if $c$ is sufficiently large,

$$
\sup _{z \in Z} \mathbf{P}\left(\left|\xi_{z}\right|>c\right)<\frac{\varepsilon}{4}
$$

\footnotetext{
${ }^{3}$ The existence of such a sequence is assured by well-known Theorem of HELLY.
} 
Since

$$
\begin{aligned}
|1-f(t, z)|= & \left|\int_{-\infty}^{\infty}\left(1-e^{i t x}\right) \mathrm{d} F(x, z)\right| \leq|t| \int_{|x| \leq c}|x| \mathrm{d} F(x, z) \\
& +2 \mathbf{P}\left(\left|\xi_{z}\right|>c\right) \leq|t| c+\frac{\varepsilon}{2}
\end{aligned}
$$

the inequality (1.9) will be satisfied whenever $|t|<\frac{\varepsilon}{2 c}=\delta$.

Now suppose that the inequality (1.9) is satisfied. Then from the inequality (1.2) we obtain $\mu_{3}<10 \varepsilon$, but this can be valid for every positive $\varepsilon$ only if $\mu_{3}=0$.

TheOREM 1.8. Suppose that for the set $\mathcal{F}=\{F(x, z), z \in Z\}$ the following two conditions are satisfied:

1. There exists a measurable function which is continuous at the point $t=0$ and for which $0 \leq g(t) \leq 1, g(0)=1$, and

$$
|f(t, z)| \geq g(t) \quad(z \in Z) .
$$

2. There exists a number $\lambda_{1}\left(0<\lambda_{1}<1\right)$ such that with a convenient choice of the quantiles $Q\left(\lambda_{1}, z\right)$, the set $\left\{Q\left(\lambda_{1}, z\right), z \in Z\right\}$ is bounded from below (from above).

In this case the set $\{Q(\lambda, z), z \in Z\}$ will be bounded from below (from above) for every $\lambda(0<\lambda<1)$.

Proof. Let us prove that under the mentioned conditions the sets $\{Q(\lambda, z), z \in Z\}$ will be bounded from below. The boundedness from above can be proved similarly. Let $Q\left(\lambda_{1}, z\right) \geq K_{1}(z \in Z)$. By means of the inequality (1.6) we get that if $\delta>0$, then

$$
\begin{aligned}
& \frac{1}{2 \delta} \int_{-\delta}^{\delta}\left(1-g^{2}(t)\right) \mathrm{d} t \geq \frac{1}{2 \delta} \int_{-\delta}^{\delta}\left(1-|f(t, z)|^{2}\right) \mathrm{d} t \\
& \quad \geq \frac{\min \left(\lambda_{1}, 1-\lambda_{1}\right)}{10} \mathbf{P}\left(\left|\xi_{z}-Q(\lambda, z)\right|>\frac{1}{\delta}\right) \geq \frac{\min \left(\lambda_{1}, 1-\lambda_{1}\right)}{10} \mathbf{P}\left(\xi_{z}<K_{1}-\frac{1}{\delta}\right),
\end{aligned}
$$

consequently

$$
\frac{\min \left(\lambda_{1}, 1-\lambda_{1}\right)}{10} \sup _{z \in Z} \mathbf{P}\left(\xi_{z}<K_{1}-\frac{1}{\delta}\right) \leq \frac{1}{2 \delta} \int_{-\delta}^{\delta}\left(1-g^{2}(t)\right) \mathrm{d} t .
$$

When $\delta \rightarrow 0$, it follows that $\mu_{1}=0$, i.e. according to the Corollary to Theorem 1.4, the set $\{Q(\lambda, z), z \in Z\}$ is bounded for every fixed $\lambda$. This completes the proof.

TheOREM 1.9. The subset $\mathcal{F}^{\prime}=\{F(x, z), z \in Z\}$ of the space $\mathcal{F}$ is compact if and only if the Condition 1 in Theorem 1.8 is satisfied and there is a number $\lambda_{1}\left(0<\lambda_{1}<1\right)$ such that the set $\left\{Q\left(\lambda_{1}, z\right), z \in Z\right\}$ is bounded by a convenient choice of the quantiles $Q\left(\lambda_{1}, z\right)$. 
Proof. Suppose the condition of our theorem to be satisfied. Then by Theorem 1.8 the set $\{Q(\lambda, z), z \in Z\}$ is bounded for every fixed $\lambda$. Hence, according to Theorem 1.5, the set $\mathcal{F}^{\prime}$ is compact.

Now let us suppose that the set $\mathcal{F}^{\prime}$ is compact. Then, according to Theorem 1.5 , the set $\{Q(\lambda, z), z \in Z\}$ will be bounded for every $\lambda$. Thus we have to show the existence of the function $g(t)$ only. For this purpose it clearly suffices to prove that the function

$$
\inf _{z \in Z}|f(t, z)|=g_{1}(t)
$$

is continuous at the point $t=0$. In contradiction to the statement let us suppose that there is a number $q<1$ and two sequences $t_{k}$ and $z_{k}$ such that $t_{k} \rightarrow 0$ if $k \rightarrow \infty$ and

$$
\left|f\left(t_{k}, z_{k}\right)\right| \leq q<1 \quad(k=1,2, \ldots) .
$$

Since the set $\mathcal{F}^{\prime}$ is compact, it follows that the sequence $F\left(x, z_{k}\right)$ contains a subsequence which converges to a distribution function $F(x)$. Without restricting the generality we can assume that the sequence $F\left(x, z_{k}\right)$ itself has this property. If $f(t)$ denotes the characteristic function of $F(x)$, then, according to what has been said, the sequence $f\left(t, z_{k}\right)$ converges uniformly to the limiting function $f(t)$ in every finite interval. But this is a contradiction because $f(t)$ is a continuous function and $f(0)=1$.

\section{$\S 5$. Some theorems concerning the convergence of a series of independent random variables}

In this paragraph we mention two theorems, the proofs of which are given in [10].

The theorems deal with the question that if $\xi_{1}, \xi_{2}, \ldots$ are independent random variables, under what conditions will the series

$$
\sum_{k=1}^{\infty} \xi_{k}
$$

converge with probability 1 regardless of the order of summation.

Let us introduce the following notations: Denote by $\mathcal{R}$ the set of finite subsets of the set of natural numbers and by $\mathcal{S}$ the set of all subsets of the set of natural numbers. Let us put

$$
\xi(A)=\sum_{k \in A} \xi_{k} \quad \text { if } \quad A \in \mathcal{R} \quad \text { or } \quad A \in \mathcal{S}
$$

assuming that the sum on the right hand side converges with probability 1 regardless of the order of summation. Denote by $F(x, A)$ the distribution function and by $Q(\lambda, A)$ an arbitrary $\lambda$-quantile of $\xi(A)$. 
Theorem 1.10. If the set $\{F(x, A), A \in \mathcal{R}\}$ is compact, then the series (1.10) converges with probability 1 regardless of the order of summation.

Conversely, if the series (1.10) converges with probability 1 regardless of the order of summation, then the set $\{F(x, a), A \in \mathcal{S}\}$ is compact.

THEOREM 1.11. If there exists a pair of numbers $\lambda_{1}, \lambda_{2}\left(0<\lambda_{1}<\lambda_{2}<1\right)$ such that by a convenient choice of the quantiles $Q\left(\lambda_{1}, A\right), Q\left(\lambda_{2}, A\right)$ the sets $\left\{Q\left(\lambda_{1}, A\right), A \in\right.$ $\mathcal{R}\},\left\{Q\left(\lambda_{2}, A\right), A \in \mathcal{R}\right\}$ are bounded, then the series (1.11) converges with probability 1 regardless of the order of summation. Conversely, if the series (1.10) converges with probability 1 regardless of the order of summation, then the sets $\{Q(\lambda, A), A \in \mathcal{S}\}$ are bounded for every fixed value of $\lambda$.

COROLlary 1. Let the distributions of the random variables $\xi_{1}, \xi_{2} \ldots$ be symmetric

with respect to the point 0 . If there exists $a \lambda \neq \frac{1}{2}(0<\lambda<1)$ such that by a convenient choice of the quantiles $Q(\lambda, A)$ the set $\{Q(\lambda, A), A \in \mathcal{R}\}$ is bounded, then the series (1.10) converges with probability 1 regardless of the order of summation.

COROLlary 2. Let the random variables $\xi_{1}, \xi_{2}, \ldots$ be non-negative. If there is a $\lambda(0<$ $\lambda<1)$ such that by a convenient choice of the quantiles $Q(\lambda, A)$ the set $\{Q(\lambda, A), A \in \mathcal{R}\}$ is bounded, then the series (1.10) converges with probability 1.

\section{ADDITIVE SET FUNCTIONS DEFINED ON RINGS AND ALGEBRAS}

In this chapter we shall analyse the properties of an additive set function $\xi(A)$ defined on the elements of a ring or algebra $\mathcal{R}$, consisting of some subsets of a set $H$. These investigations will help us to find out under which conditions we can extend the set function $\xi(A)$ to the smallest $\sigma$-ring containing the ring (or algebra) $\mathcal{R}$. It is clear that one of the conditions necessary for this is the complete additiveness of $\xi(A)$ over $\mathcal{R}$. Therefore we have to find first a condition that ensures that an additive set function should be completely additive. This question will be answered by

THEOREM 2.1. In order that an additive set function $\xi(A)$ defined on the elements of the ring $\mathcal{R}$ should be completely additive it is necessary and sufficient that for every non-decreasing sequence of sets $B_{1}, B_{2}, \ldots$ with $B_{k} \in \mathcal{R}(k=1,2, \ldots) \prod_{k=1}^{\infty} B_{k}=0$, the following condition holds:

$$
\mathbf{P}\left(\left|\xi\left(B_{k}\right)\right|>\varepsilon\right) \rightarrow 0 \quad \text { if } \quad k \rightarrow \infty,
$$

where $\varepsilon$ is an arbitrary positive number.

Proof. Suppose that the condition (2.1) is satisfied. Let $A_{1}, A_{2}, \ldots$ be a sequence of disjoint sets of the ring $\mathcal{R}$. Let $A=\sum_{k=1}^{\infty} A_{k}, B_{n}=\sum_{k=n}^{\infty} A_{k}$. 
Since $\xi(A)$ is an additive set function, it follows that

$$
\xi(A)=\sum_{k=1}^{n-1} \xi\left(A_{k}\right)+\xi\left(B_{n}\right) \quad(n=2,3, \ldots) .
$$

Hence

$$
\xi(A)-\sum_{k=1}^{n-1} \xi\left(A_{k}\right)=\xi\left(B_{n}\right) \Rightarrow 0 \quad \text { if } \quad n \rightarrow \infty,
$$

consequently ([4], p. 119, Corollary 2)

$$
\xi(A)=\sum_{k=1}^{\infty} \xi\left(A_{k}\right)
$$

Now let us suppose that $\xi(A)$ is completely additive. If $B_{1}, B_{2}, \ldots$ is a sequence of sets having the properties mentioned in Theorem 2.1 and $A_{n}=B_{n}-B_{n+1}(n=1,2, \ldots)$, then $A_{i} A_{k}=0$ if $i \neq k$ and

$$
B_{1}=\sum_{k=1}^{\infty} A_{k}
$$

hence, by the complete additiveness of $\xi(A)$, it follows that

$$
\xi\left(B_{1}\right)=\sum_{k=1}^{\infty} \xi\left(A_{k}\right)
$$

Thus we obtain that the following relation holds:

$$
\xi\left(B_{n}\right)=\sum_{k=n}^{\infty} \xi\left(A_{k}\right) \rightarrow 0 \quad \text { if } \quad n \rightarrow \infty
$$

consequently, it is also true that if $\varepsilon>0$, then

$$
\mathbf{P}\left(\left|\xi\left(B_{n}\right)\right|>\varepsilon\right) \rightarrow 0
$$

Corollary. Let $\xi(A)$ be an additive set function defined on the ring $\mathcal{R}$. If there exists a positive number $T$ such that for every non-increasing sequence $B_{1}, B_{2}, \ldots$ of sets belonging to $\mathcal{R}$ for which $\lim _{k \rightarrow \infty} B_{k}=0$, we have

$$
\lim _{k \rightarrow \infty} f\left(t, B_{k}\right)=1 \quad \text { if } \quad|t| \leq T,
$$

then the set function $\xi(A)$ is completely additive. 
Proof. From the inequality (1.3), valid for every characteristic function, it follows that the relation

$$
\lim _{k \rightarrow \infty} f\left(T, B_{k}\right)=1
$$

holds for every $t$, i.e.

$$
\xi\left(A_{k}\right) \Rightarrow 0 \quad \text { if } \quad k \rightarrow \infty .
$$

The statement then follows from Theorem 2.1.

In what follows some theorems concerning the quantiles $Q(\lambda, A)$ of the random variables $\xi(A)$ will be proved. We shall make use of the results of the preceding chapter.

TheOREm 2.2. Let $\xi(A)$ be an additive set function defined on an algebra $\mathcal{R}$. Suppose that there is a number $\lambda_{1}$ such that by a convenient choice of the quantiles $Q\left(\lambda_{1}, A\right)$ the set $\left\{Q\left(\lambda_{1}, A\right), A \in \mathcal{R}\right\}$ is bounded form below (from above). Then, for every $\lambda$, the set $\{Q(\lambda, A), A \in \mathcal{R}\}$ is bounded from below (from above). If the set $\left\{Q\left(\lambda_{1}, A\right) A \in \mathcal{R}\right\}$ is bounded from both sides, then the set $\{F(x, A), A \in \mathcal{R}\}$ is compact.

Proof. If $A \in \mathcal{R}$, then, by assumption, $\bar{A} \in \mathcal{R}$ and $H=A+\bar{A} \in R$. Hence it follows that

$$
\xi(H)=\xi(A)+\xi(\bar{A}), \quad f(t, H)=f(t, A) f(t, \bar{A}) .
$$

Since $|f(t, A)| \leq 1$, it follows that

$$
|f(t, A)| \geq|f(t, H)| \quad(A \in \mathcal{R}) .
$$

$f(t, H)$ is a characteristic function; consequently $f(t, H)$ is continuous, and $f(0, H)=1$. Hence, if

$$
g(t)=|f(t, H)|
$$

we obtain that Condition 1 of Theorem 1.8 is satisfied. As the fulfilment of Condition 2 has been supposed one part of the statement is proved.

If we suppose that the set $\left\{Q\left(\lambda_{1}, A\right), A \in \mathcal{R}\right\}$ is bounded from both sides, then from inequality (2.2), further from Theorem 1.9 it follows that the set $\{F(x, A), A \in \mathcal{R}\}$ is compact. This completes the proof of the theorem.

If $\mathcal{R}$ is a ring, the existence of a quantile-set bounded from both sides does not imply that every quantile-set is bounded. If e.g. $\mathcal{R}$ is the ring formed by the finite sets of positive integers and

$$
F(x, n)=\frac{1}{\sqrt{2 \pi}} \int_{-\infty}^{\infty} e^{-\frac{t^{2}}{2}} \mathrm{~d} t
$$

then $Q\left(\frac{1}{2}, A\right)=0$ if $A \in \mathcal{R}$. However, the quantities $Q(\lambda, A)$ are not bounded. In case of a ring we obtain, using the results of Chapter I, the following theorems. 
TheOREm 2.3. Let $\xi(A)$ be an additive set function defined on a ring $\mathcal{R}$. Suppose that there is a pair of numbers $\lambda_{1}, \lambda_{2}\left(0<\lambda_{1}<\lambda_{2}<1\right)$ such that by a convenient choice of the quantiles $Q\left(\lambda_{1}, A\right), Q\left(\lambda_{2}, A\right)$ the sets $\left\{Q\left(\lambda_{1}, A\right), A \in \mathcal{R}\right\},\left\{Q\left(\lambda_{2}, A\right), A \in \mathcal{R}\right\}$ are bounded. In this case the set $\{Q(\lambda, A), A \in \mathcal{R}\}$ is bounded for every fixed $\lambda(0<\lambda<1)$.

Proof. Contrarily to the statement of the theorem let us suppose that there is a number $\lambda_{0}$ and a sequence of sets $A_{k}$ such that by a convenient choice of the quantiles $Q\left(\lambda_{0}, A_{k}\right)$ we have $\left|Q\left(\lambda_{0}, A_{k}\right)\right| \rightarrow \infty$ if $k \rightarrow \infty$. Let $B_{n}=\sum_{k=1}^{n} A_{k}, C_{n}=B_{n+1}-B_{n}$. Then $C_{i} C_{k}=0$ if $i \neq k$. Hence the random variables $\xi\left(C_{n}\right)$ are independent. The conditions of our theorem and Theorem 1.11 together imply the convergence with probability 1 of the series

$$
\sum_{n=1}^{\infty} \xi\left(C_{n}\right)
$$

Denote by $\xi$ the sum of this series and by $f(t)$ the characteristic function of $\xi$. It is clear that

$$
f(t)=f\left(t, A_{1}+A_{2}+\cdots+A_{n}\right) \prod_{k=n}^{\infty} f\left(t, C_{k}\right),
$$

further that

$$
f\left(t, A_{1}+A_{2}+\cdots+A_{n}\right)=f\left(t,\left(A_{1}+A_{2}+\cdots A_{n}\right)-A_{n}\right) f\left(t, A_{n}\right),
$$

hence

$$
|f(t)| \leq\left|f\left(t, A_{1}+A_{2}+\cdots+A_{n}\right)\right| \leq\left|f\left(t, A_{n}\right)\right| .
$$

We know that the set $\left\{Q\left(\lambda_{1}, A\right), A \in \mathcal{R}\right\}$ is bounded; hence, by Theorems 1.9 and 1.5 it follows that the quantiles of the random variables $\xi\left(A_{n}\right)$ are bounded, but this is a contradiction because we have supposed that $\left|Q\left(\lambda_{0}, A_{n}\right)\right| \rightarrow \infty$ if $n \rightarrow \infty$.

THEOREM 2.4. Let $\xi(A)$ be an additive set function defined on a ring $\mathcal{R}$. Suppose that $\xi(A) \geq 0(A \in \mathcal{R})$ and there is a number $\lambda_{1}$ such that by a convenient choice of the quantiles $Q\left(\lambda_{1}, A\right)$ the set $\left\{Q\left(\lambda_{1}, A\right), A \in \mathcal{R}\right\}$ is bounded. In this case the sets $\{Q(\lambda, A), A \in \mathcal{R}\}$ will be bounded for every fixed $\lambda(0<\lambda<1)$.

Proof. Contrarily to our statement let us suppose that there is a number $\lambda_{0}$ and a sequence of sets $A_{k}$ such that by a convenient choice of the quantiles $Q\left(\lambda_{0}, A_{k}\right)$ we have $Q\left(\lambda_{0}, A_{k}\right) \rightarrow \infty$ if $k \rightarrow \infty$. If $B_{n}=\sum_{k=1}^{n} A_{k}$, then $B_{n+1} \supseteq B_{n}, B_{n} \supseteq A_{n}$. Since $\xi(A)$ is additive and non-negative, we have $\xi\left(B_{n}\right) \geq \xi\left(A_{n}\right)$. If $C_{n}=B_{n+1}-B_{n}$, then $C_{i} C_{k}=0$ if $i \neq k$, consequently the variables $\xi\left(C_{n}\right)$ are independent. We know further that if $A \in \mathcal{R}$, then the quantities $Q\left(\lambda_{1}, A\right)$ are bounded, hence by Corollary 2 of Theorem 1.11 we obtain

$$
\sum_{n=1}^{\infty} \xi\left(C_{n}\right)<\infty
$$


If we denote the sum of this series by $\xi$, then

$$
0 \leq \xi\left(A_{n}\right) \leq \xi\left(B_{n}\right)=\sum_{k=1}^{n} \xi\left(C_{k}\right) \leq \xi
$$

but this is a contradiction because we have supposed that $Q\left(\lambda_{0}, A_{k}\right) \rightarrow \infty$. Thus our theorem is proved.

Let $\mathcal{R}$ be a ring and $\xi(A)$ a set function defined on the elements of $\mathcal{R}$. Let us define the following set functions:

$$
\begin{aligned}
& \mu_{1}(A)=\lim _{\varepsilon \rightarrow \infty} \sup _{B \in A \mathcal{R}} \mathbf{P}(\xi(B)<-\varepsilon), \\
& \mu_{2}(A)=\lim _{\varepsilon \rightarrow \infty} \sup _{B \in A \mathcal{R}} \mathbf{P}(\xi(B)>\varepsilon), \\
& \mu_{3}(A)=\lim _{\varepsilon \rightarrow \infty} \sup _{B \in A \mathcal{R}} \mathbf{P}(|\xi(B)|>\varepsilon),
\end{aligned}
$$

The set functions $\mu_{1}(A), \mu_{2}(A), \mu_{3}(A)$ are the same as the quantities $\mu_{1}, \mu_{2}, \mu_{3}$ defined in Chapter I, provided that $Z=A \mathcal{R}$. It is clear that if $B \in A \mathcal{R}, A \in \mathcal{R}$, then

$$
\mu_{i}(B) \leq \mu_{i}(A) \quad(i=1,2,3) .
$$

In addition there holds the following

THEOREM 2.5. If $\xi(A)$ is an additive set function defined on the elements of a ring $\mathcal{R}$, and $A_{k}(k=1,2, \ldots, r)$ is a finite sequence of sets belonging to $\mathcal{R}$, then

$$
\mu_{i}\left(\sum_{k=1}^{r} A_{k}\right) \leq \sum_{k=1}^{r} \mu_{i}\left(A_{k}\right) \quad(i=1,2,3) .
$$

Proof. Let us carry out the proof in the case $i=3$. The other cases can be treated in a similar way. Let $A_{k} \in \mathcal{R}, A_{k}^{\prime} \in A_{k} \mathcal{R}(k=1,2, \ldots, r), A_{i} A_{l}=0$ if $i \neq l$, then we have

$$
\begin{gathered}
\mathbf{P}\left(\left|\xi\left(A_{1}^{\prime}+A_{2}^{\prime}+\cdots+A_{r}^{\prime}\right)\right|>\varepsilon\right)=\mathbf{P}\left(\left|\xi\left(A_{1}^{\prime}\right)+\xi\left(A_{2}^{\prime}\right)+\cdots+\xi\left(A_{r}^{\prime}\right)\right|>\varepsilon\right) \\
\leq \mathbf{P}\left(\left|\xi\left(A_{1}^{\prime}\right)\right|>\frac{\varepsilon}{r}\right)+\mathbf{P}\left(\left|\xi\left(A_{2}^{\prime}\right)\right|>\frac{\varepsilon}{r}\right)+\cdots+\mathbf{P}\left(\left|\xi\left(A_{r}^{\prime}\right)\right|>\frac{\varepsilon}{r}\right) .
\end{gathered}
$$

Consequently

$$
\sup _{C \in \sum_{k=1}^{r} A_{k} \mathcal{R}} \mathbf{P}(|\xi(C)|>\varepsilon) \leq \sum_{k=1}^{r} \sup _{A_{k}^{\prime} \in A_{k} \mathcal{R}} \mathbf{P}\left(\left|\xi\left(A_{k}^{\prime}\right)\right|>\frac{\varepsilon}{r}\right),
$$

hence it follows that

$$
\mu_{3}\left(\sum_{k=1}^{r} A_{k}\right) \leq \sum_{k=1}^{r} \mu_{3}\left(A_{k}\right)
$$


Now, if $A_{1}, A_{2}, \ldots, A_{r}$ are arbitrary sets belonging to the ring $\mathcal{R}$, then, according to the previous inequalities, we have

$$
\mu_{3}\left(\sum_{k=1}^{r} A_{k}\right) \leq \mu_{3}\left(A_{1}\right)+\mu_{3}\left(A_{2}-A_{1}\right)+\cdots+\mu_{3}\left(A_{r}-\sum_{k=1}^{r-1} A_{k}\right) .
$$

Since the set functions $\mu_{i}(A)(i=1,2,3)$ are monotonous, it follows that

$$
\mu_{3}\left(\sum_{k=1}^{r} A_{k}\right) \leq \sum_{k=1}^{r} \mu_{3}\left(A_{k}\right) .
$$

This completes the proof of our statement.

Regarding the set functions $\mu_{i}(A)$ a law of 0 or 1 holds. This will be expressed by

TheOREM 2.6. The set functions $\mu_{1}(A), \mu_{2}(A), \mu_{3}(A)$ defined on the elements of the ring $\mathcal{R}$ can take on only the values 0 or 1 .

Proof. Let $A \in \mathcal{R}$ and consider those elements of the ring $\mathcal{R}$ for which $B \subseteq A$ holds. If $\mu_{1}(A)>0\left(\mu_{2}(A)>0\right)$, then by the Corollary of Theorem 1.4 there is a $\lambda_{1}\left(\lambda_{2}\right)$ such that by a convenient choice of the quantiles $Q\left(\lambda_{1}, B\right)\left(Q\left(\lambda_{2}, B\right)\right)$, the set $\left\{Q\left(\lambda_{1}, B\right), B \in A \mathcal{R}\right\}\left(\left\{Q\left(\lambda_{2}, B\right), B \in A \mathcal{R}\right\}\right)$ is not bounded from below (from above). Then, using Theorem 2.2, it follows that also the sets $\{Q(\lambda, B), B \in A \mathcal{R}\}$ are not bounded from below (from above) for every fixed value of $\lambda$. Hence, using again the Corollary of Theorem 1.4, we get $\mu_{1}(A)=1\left(\mu_{2}(A)=1\right)$. Finally, let us consider the value of $\mu_{3}(A)$. If $\mu_{3}(A)>0$, then, from $\mu_{3}(A) \leq \mu_{1}(A)+\mu_{2}(A)$, it follows that at least one of $\mu_{1}(A)$ and $\mu_{2}(A)$ is positive. Taking into account what has been said above and the fact that $\mu_{3}(A) \geq \mu_{1}(A), \mu_{3}(A) \geq \mu_{2}(A)$, it follows that $\mu_{3}(A)=1$. This completes the proof.

Finally let us prove

THEOREM 2.7. Let $\xi(A)$ be an additive (completely additive) set function defined on a ring $\mathcal{R}$. Then the set function $|1-f(t, A)|$ is subadditive (completely subadditive) for every fixed $t$.

Proof. Let $A_{1}, A_{2}, \ldots$ be a sequence of disjoint sets of the ring $\mathcal{R}$. By the inequality

$$
\left|1-z_{1} z_{2} \cdots z_{r}\right| \leq\left|1-z_{1}\right|+\left|1-z_{2}\right|+\cdots+\left|1-z_{r}\right|
$$

valid for such complex numbers $z_{i}$ for which $\left|z_{i}\right| \leq 1(i=1,2, \ldots)$, we obtain that

$$
\left|1-f\left(t, \sum_{k=1}^{r} A_{k}\right)\right|=\left|1-\prod_{k=1}^{r} f\left(t, A_{k}\right)\right| \leq \sum_{k=1}^{r}\left|1-f\left(t, A_{k}\right)\right| \quad(r=1,2, \ldots) .
$$


If $\xi(A)$ is additive, the statement is proved. If $\xi(A)$ is completely additive and $A=$ $\sum_{k=1}^{\infty} A_{k} \in \mathcal{R}$, then, by virtue of

$$
f\left(t, \sum_{k=1}^{r} A_{k}\right)=\prod_{k=1}^{r} f\left(t, A_{k}\right) \Rightarrow f(t, A),
$$

it follows that

$$
|1-f(t, A)| \leq \sum_{k=1}^{\infty}\left|1-f\left(t, A_{k}\right)\right| .
$$

This completes the proof.

\section{EXTENSION OF COMPLETELY ADDITIVE SET FUNCTIONS}

\section{$\S 1$. The discussion of the problem}

Let $\mathcal{R}$ be a ring consisting of certain subsets of a space $H$ and $\xi(A)$ a completely additive set function defined on the elements of $\mathcal{R}$. In this chapter we shall deal with the question under what conditions can the set function $\xi(A)$ be extended to the $\sigma$-ring $\mathcal{S}(\mathcal{R})$. We mean by extension the construction of a completely additive set function $\xi^{*}(A)$ defined on the elements of $\mathcal{S}(\mathcal{R})$ for which

$$
\xi^{*}(A)=\xi(A) \quad \text { if } \quad A \in \mathcal{R} .
$$

It is clear that not every completely additive set function can be extended; if, in particular, $\xi(\omega, A)$ is constant, $\xi(\omega, A) \equiv \varphi(A)$ where $\varphi(A)$ is a real-valued, completely additive set function defined on the elements of $\mathcal{R}$, then we need also further conditions for its extension.

Let $H$ be e.g. the square $0 \leq x<1,0 \leq y<1$ on the $(x, y)$-plane and $\mathcal{R}$ be the algebra formed 1 . by the finite sums of intervals, closed to the left, open to the right and lying on straight lines parallel to the $x$-axis, 2 . by the complements of the previous sets (these sets will be shortly called to be of complementary type) and 3 . by the set $H$ itself. If $A=\sum_{k=1}^{n}\left\{a_{k} \leq x<b_{k}, y=y_{k}\right\}$, then put $\varphi(A)=\sum_{k=1}^{n}\left(b_{k}-a_{k}\right)$ and $\varphi(\bar{A})=-\varphi(A) . \varphi(A)$ is a completely additive set function. In fact, if $A_{1}, A_{2}, \ldots$ is a sequence of disjoint sets of $\mathcal{R}$ and $A=\sum_{k=1}^{\infty} A_{k} \in \mathcal{R}$, then there will be at most one set among the sets $A_{1}, A_{2}, \ldots$ which is of complementary type. If there is none, then $A$ is the sum of a finite number of intervals closed to the left and open to the right, such that each of them is divided into a sum of intervals of the same type. In whatever order we add the lengths of them we always get the same sum, i.e. $\varphi(A)=\sum_{k=1}^{\infty} \varphi\left(A_{k}\right)$. But if we have a 
set of complementary type and this is $A_{1}$, then from $\varphi(A)=\varphi\left(A_{1}\right)+\varphi\left(\sum_{k=2}^{\infty} A_{k}\right)$ and $\varphi\left(\sum_{k=2}^{\infty} A_{k}\right)=\sum_{k=2}^{\infty} \varphi\left(A_{k}\right)$ it follows that $\varphi(A)=\sum_{k=1}^{\infty} \varphi\left(A_{k}\right)$. In spite of this, $\varphi(A)$ can not be extended to $\mathcal{S}(\mathcal{R})$, because the values $+\infty$ and $-\infty$ can not occur simultaneously in the set of values of a completely additive set function defined on a $\sigma$-ring ([6], p. 18, Theorem 3.4.13).

In case of ordinary real-valued set functions it is known that in order to be able to carry out the extension and to have an extended set function of finite value it is necessary and sufficient that the original set function should be bounded from both sides. The extension can be carried out most simply by representing the set function as the difference of two non-negative, completely additive set functions and by extending both these separately.

In case of random-valued set functions this way is inpracticable, namely if the set of the measurable subsets of the set $A$ is not countable, then the functions

$$
\eta^{+}(A)=\sup _{B \in A \mathcal{R}} \xi(B), \quad \eta^{-}(A)=\inf _{B \in A \mathcal{R}} \xi(B)
$$

defined on the space of the elementary events are not certainly measurable; admitted that they are measurable, it is again possible that they have an infinite value on a set of positive measure. Therefore we have to look for another way of extension in this case.

We have to emphasize that in the particular case $\xi(\omega, A) \equiv \varphi(A)$ the requirement of $\xi(\omega, A)$ being a random variable means that the value $\varphi(A)$ is finite. Consequently, from the real-valued set functions we obtain as particular cases of random set functions only the finite-valued ones.

There arises the question whether we can construct the whole theory of random-valued set functions starting from the family of sample functions (which should be common real-valued set functions). This way is suitable only if we are satisfied with additive set functions. Namely, if we require the sample functions to be completely additive, then we have to exclude several important set functions from the investigations. E.g. let $\xi_{t}(t \geq 0)$ be the well-known Brownian movement process with $\mathbf{M}\left(\xi_{t}\right)=0, \mathbf{D}^{2}\left(\xi_{t}\right)=t$. If $\mathcal{R}$ consists of finite sums of intervals of the real axis, which are closed to the left and open to the right and the set function $\xi(A)$ is an additive set function formed by the corresponding differences and sums of differences, respectively, of the process, then the sample functions $\omega(A)$ of the set function $\xi(A)$ can not be completely additive. This can be seen easily. Let us restrict ourselves to the interval $I=[0,1)$, i.e. suppose $A \subseteq I$. Let $\tilde{\xi}_{t}$ be a separable Brownian movement process defined in the interval $0 \leq t \leq 1$ such that

$$
\mathbf{P}\left(\tilde{\xi}_{t}=\xi_{t}\right)=1
$$

and let $\tilde{\xi}(A)$ be the additive set function generated by the differences and by the sums of differences, respectively, of the process $\tilde{\xi}_{t}$. It is clear that

$$
\mathbf{P}(\tilde{\xi}(A)=\xi(A))=1 \quad \text { if } \quad A \in \mathcal{R} .
$$


Almost every sample function of the process $\tilde{\xi}_{t}$ is continuous ([4], p. 393, Theorem 2.2). Hence and by Theorem 2.3 of [4] (p. 395) it follows that almost every sample function of a separable Brownian movement process is not of bounded variation. Let us divide the interval $I=[0,1)$ into a sum of disjoint intervals $\mathcal{J}_{1}, \mathcal{J}_{2}, \ldots, \mathcal{J}_{n}$ of length $\frac{1}{n}$, closed to the left, open to the right and put

$$
\tilde{\zeta}=\sum_{k=1}^{n}\left|\tilde{\xi}\left(\mathcal{J}_{k}\right)\right|, \quad \zeta_{n}=\sum_{k=1}^{n}\left|\xi\left(\mathcal{J}_{k}\right)\right| .
$$

From what has been said above it follows that

$$
\mathbf{P}\left(\tilde{\zeta}_{n} \rightarrow \infty\right)=1 .
$$

Since $\mathbf{P}\left(\tilde{\zeta}_{n}=\zeta_{n}\right)=1$, we have

$$
\mathbf{P}\left(\zeta_{n} \rightarrow \infty\right)=1
$$

i.e. almost every sample function of the set function $\xi(A)$ is not of bounded variation.

The results of this chapter will help us to solve existence problems of additive set functions $\xi(A)$ defined on Borel sets of the space $H$. We shall consider first, to make clear the idea, a concrete case.

Let us construct e.g. a set function $\xi(A)$, defined on the set $\mathcal{R}$ of Borel subsets of some finite closed $n$-dimensional interval $H$ of the space $\mathcal{R}_{n}$, such that $\xi(A)$ has for any $A \in \mathcal{R}$ a Cauchy distribution; the characteristic function of the random variable $\xi(A)$ will be

$$
f(t, A)=e^{-|A||t|} .
$$

First of all by aid of the construction due to Kolmogorov $([8], \S 4)$ we construct a family of random variables $\xi_{t_{1}, t_{2}, \ldots, t_{n}}\left(t_{1}, t_{2}, \ldots, t_{n}\right) \in H$ for which the following conditions hold:

a) If $I$ is the $n$-dimensional interval

$$
I=\left\{a_{i} \leq x_{i}<a_{i}+h_{i} ; \quad i=1,2, \ldots, n\right\}, \quad I \subseteq H
$$

and

$$
\Delta_{h_{i}} \xi_{t_{1}, t_{2}, \ldots, t_{n}}=\xi_{t_{1}, \ldots, t_{i-1}, t_{i}+h_{i}, t_{i+1}, \ldots, t_{n}}-\xi_{t_{1}, t_{2}, \ldots, t_{n}},
$$

further

$$
\Delta_{I} \xi_{t_{1}, t_{2}, \ldots, t_{n}}=\Delta_{h_{1}} \Delta_{h_{2}} \ldots \Delta_{h_{n}} \xi_{t_{1}, t_{2}, \ldots, t_{n}}
$$

then the random variable

$$
\xi(I)=\Delta_{I} \xi_{t_{1}, t_{2}, \ldots, t_{n}}
$$

has a Cauchy distribution, namely

$$
f(t, I)=e^{-h_{1} h_{2} \ldots h_{n}|t|} .
$$


b) If $I_{1}, I_{2}, \ldots, I_{r}$ are $n$-dimensional disjoint intervals lying in the interval $H$, then the random variables

$$
\xi\left(I_{1}\right), \xi\left(I_{2}\right), \ldots, \xi\left(I_{r}\right)
$$

defined by expression (3.2) are independent.

We consider the ring $\mathcal{R}$ formed by the finite sums of the interval (3.1) and let the random variables

$$
\xi(A)=\sum_{k=1}^{r} \xi\left(I_{k}\right)
$$

correspond to its elements $A=I_{1}+I_{2}+\cdots+I_{r}, I_{l} \subseteq H(l=1,2, \ldots, r), I_{j} I_{l}=0$ if $j \neq l$.

Having arrived so far through Kolmogorov's construction, we state that $\xi(A)$ is completely additive on the ring $\mathcal{R}$, further the set $\{F(x, A), A \in \mathcal{R}\}$ is compact. Then, taking Theorem 3.2 into account, we carry out the extension of the set function $\xi(A)$ to the $\sigma$-ring $\mathcal{S}(\mathcal{R})$ of the Borel sets of the set $H .^{4}$

The complete additiveness of $\xi(A)$ follows from Theorem 2.1 and from that $\left|A_{n}\right| \rightarrow 0$ if $A_{n} \rightarrow 0$. Namely, by (3.3) and (3.4) we obtain

$$
f\left(t, A_{n}\right)=e^{-\left|A_{n}\right||t|} \Rightarrow 0 \quad \text { if } \quad n \rightarrow \infty .
$$

The second property, the compactness of the set $\{F(x, A), A \in \mathcal{R}\}$ follows from

$$
f(t, A)=e^{-|A||t|} \geq e^{-|H||t|}=g(t),
$$

where $g(t)$ is a continuous function, $0 \leq g(t) \leq 1, g(0)=1$. As $Q\left(\frac{1}{2}, A\right)=0$, the conditions of Theorem 1.9 are satisfied. By this step the construction is finished.

\section{$\S 2$. Extension of a set function defined on a ring}

TheOREm 3.1. Let $\xi(A)$ be a completely additive set function defined on a ring $\mathcal{R}$. If there is a positive number $T$ such that for every fixed value of $t$ for which $|t| \leq T$ the set function $|1-f(t, A)|$ is of bounded variation, then $\xi(A)$ can be extended to the $\sigma$-ring $\mathcal{S}(\mathcal{R})$. The extension is unique in the sense that if $\xi^{*}(A)$ and $\xi^{* *}(A)$ are completely additive set functions defined on the $\sigma$-ring $\mathcal{S}(\mathcal{R})$ and

$$
\xi^{*}(A)=\xi^{* *}(A) \quad \text { if } \quad A \in \mathcal{R},
$$

then

\footnotetext{
${ }^{4}$ In the present case $\mathcal{S}(\mathcal{R})$ is not only a $\sigma$-ring, but also a $\sigma$-algebra.
} 


$$
\xi^{*}(A)=\xi^{* *}(A) \quad \text { if } \quad A \in \mathcal{S}(\mathcal{R}) .
$$

Conversely, if $\xi(A)$ is a completely additive set function defined on a $\sigma$-ring $\mathcal{S}$ and $T$ is an arbitrary positive number, then the set function

$$
\sup _{|t| \leq T}|1-f(t, A)|
$$

is of bounded variation.

Proof of THE FIRST PART of THE THEOREM. By assumption $\xi(A)$ is a completely additive set function on the ring $\mathcal{R}$. Now, if $A_{1}, A_{2}, \ldots$ is a sequence of disjoint sets of the $\operatorname{ring} \mathcal{R}, A=\sum_{k=1}^{\infty} A_{k} \in \mathcal{R}$, then

$$
\lim _{n \rightarrow \infty} \sup _{|t| \leq T}\left|f(t, A)-\prod_{k=1}^{n} f\left(t, A_{k}\right)\right|=0 .
$$

Let us consider the Banach-algebra $\mathcal{B}$ of continuous functions in the interval $[-T, T]$; let the maximum of the absolute value of a function be its norm. It is clear that $\mathcal{B}$ is commutative and has a unit element. By (3.6), if we consider the characteristic functions, defined on the ring $\mathcal{R}$, only in the interval $[-T, T]$ and put $g(t, A)=f(t, A)$ if $|t| \leq T$, then $g(t, A)$ will be a completely multiplicative ${ }^{5}$ set function defined on the ring $\mathcal{R}$ whose values belong to the Banach algebra $\mathcal{B}$. We note that in virtue of $g(0, A)=1$, for $A \in \mathcal{R}$, the values of the set function $g(t, A)$ are different from 0 .

We prove that the set function $\|1-g(t, A)\|(A \in \mathcal{R})$ is of bounded variation and completely subadditive.

In order to prove the first statement we show that the conditions of Theorem 1.1 hold. Since $\|g(t, A)\| \leq 1$, consequently $\|1-g(t, A)\| \leq 2$, hence Condition 1 is satisfied.

According to Theorem 2.7 if $A_{1}, A_{2}, \ldots, A_{r}$ are disjoint sets of the ring $\mathcal{R}, A=\sum_{i=1}^{r} A_{i}$, then for every value of $t$

$$
|1-f(t, A)| \leq \sum_{k=1}^{r}\left|1-f\left(t, A_{k}\right)\right|
$$

i.e.

$$
\|1-g(t, A)\|=\sup _{|t| \leq T}|1-f(t, A)| \leq \sum_{k=1}^{r} \sup _{t \leq T}\left|1-f\left(t, A_{k}\right)\right|=\sum_{k=1}^{r}\left\|1-g\left(t, A_{k}\right)\right\| .
$$

\footnotetext{
${ }^{5}$ The definition of this notion may be found in the paper [11].
} 
Consequently, Condition 2 is also satisfied. Let $A_{1}, A_{2}, \ldots$ be a sequence of disjoint sets of the ring $\mathcal{R}$. According to the condition of our theorem

$$
\sum_{k=1}^{\infty}\left|1-f\left(t, A_{k}\right)\right|<\infty \quad \text { if } \quad|t| \leq T .
$$

Hence it follows ([4], p. 115, Theorem 2.7) that every rearrangement of the series $\sum_{k=1}^{\infty} \xi\left(A_{k}\right)$ converges with probability 1 . On the other hand, this involves the absolute convergence of the infinite series

$$
\sum_{k=1}^{\infty} \int_{|x| \leq \varepsilon} x \mathrm{~d} F\left(x, A_{k}\right), \quad \sum_{k=1}^{\infty} \int_{|x| \leq \varepsilon} x^{2} \mathrm{~d} F\left(x, A_{k}\right), \quad \sum_{k=1}^{\infty} \mathbf{P}\left(\left|\xi\left(A_{k}\right)\right|>\varepsilon\right)
$$

$([8], \S 5)$.

According to what has been said above it follows from the inequality

$$
\begin{aligned}
\left\|1-g\left(t, A_{k}\right)\right\|= & \sup _{|t| \leq T}\left|1-f\left(t, A_{k}\right)\right| \leq \sup _{|t| \leq T} \mid \int_{|x| \leq \varepsilon}\left(e^{i t x}-1-i t x\right) \mathrm{d} F\left(x, A_{k}\right) \\
& +i t \int_{|x| \leq \varepsilon} x \mathrm{~d} F\left(x, A_{k}\right) \mid+2 \mathbf{P}\left(\left|\xi\left(A_{k}\right)\right|>\varepsilon\right) \\
\leq & \frac{T^{2}}{2} \int_{|x| \leq \varepsilon} x^{2} \mathrm{~d} F\left(x, A_{k}\right)+T\left|\int_{|x| \leq \varepsilon} x \mathrm{~d} F\left(x, A_{k}\right)\right|+2 \mathbf{P}\left(\left|\xi\left(A_{k}\right)\right|>\varepsilon\right)
\end{aligned}
$$

that

$$
\sum_{k=1}^{\infty}\left\|1-g\left(t, A_{k}\right)\right\|<\infty
$$

i.e. Condition 3 of Theorem 1.1 is also satisfied.

The second statement follows immediately from Theorem 2.7; namely, in the set function $|1-f(t, A)|$ is completely subadditive for every value of $t$, then the set function $\|1-g(t, A)\|=\sup _{|t| \leq T}|1-f(t, A)|$ is also completely subadditive.

We have thus proved that the conditions of Theorem 1 in [11] are satisfied; consequently, the set function $g(t, A)$ can be uniquely extended to the $\sigma$-ring $\mathcal{S}(\mathcal{R})$. By other words, there exists one and only one completely multiplicative set function $g^{*}(t, A)$, defined on the $\sigma$-ring $\mathcal{S}(\mathcal{R})$, for which

and

$$
g^{*}(t, A)=g(t, A) \quad \text { if } \quad A \in \mathcal{R}
$$

$$
g^{*}(t, A) \in \mathcal{B} \quad \text { if } \quad A \in \mathcal{S}(\mathcal{R}) .
$$


Further, it is true that if $A_{n} \in \mathcal{S}(\mathcal{R})(n=1,2, \ldots)$ and $\lim _{n \rightarrow \infty} A_{n}=A$, then $\lim _{n \rightarrow \infty} g^{*}\left(t, A_{n}\right)=g^{*}(t, A)$. In particular, if $A=0$, then the equality $g^{*}(t, 0)=1$ implies that $\lim _{n \rightarrow \infty} g^{*}\left(t, A_{n}\right)=1$.

The extension of the set function $\xi(A)$ will be carried out by transfinite induction.

Let us construct a transfinite sequence of classes of sets $\mathcal{R}_{0}=\mathcal{R}, \mathcal{R}_{1}, \mathcal{R}_{2}, \ldots$ as follows. If for every number $\nu$ for which $\nu<\nu^{\prime}<\omega_{1}$ the class of sets $\mathcal{R}_{\nu}$ has been already defined, then let $\mathcal{R}_{\nu^{\prime}}$ be the system of the sets which can be obtained as limits of convergent sequences of sets belonging to the class $\sum_{\nu<\nu^{\prime}} \mathcal{R}_{\nu}$. It is clear that every class $\mathcal{R}_{\nu}\left(\nu<\omega_{1}\right)$ is a ring.

Suppose that there are already corresponding random variables to the elements of every ring $\mathcal{R}_{\nu}$ where $\nu<\nu^{\prime}<\omega_{1}$; more exactly suppose that for every $\nu<\nu^{\prime}$ we have defined on the elements of $\mathcal{R}_{\nu}$ a completely additive set function $\xi_{\nu}(A)$ for which the relations

$$
\begin{array}{ccc}
\xi_{\nu}(A)=\xi_{\nu_{0}}(A) \quad \text { if } \quad \nu_{0}<\nu, & A \in \mathcal{R}_{\nu_{0}}, \\
f_{\nu}(t, A)=g^{*}(t, A) \quad \text { if } & |t| \leq T, \quad A \in \mathcal{R}_{\nu}
\end{array}
$$

are satisfied where $f_{\nu}(t, A)$ is the characteristic function of the random variable $\xi_{\nu}(A)$. Now we start to define the set function $\xi_{\nu^{\prime}}(A)\left(A \in \mathcal{R}_{\nu^{\prime}}\right)$.

First we show that if $A_{1}, A_{2}, \ldots$ is a convergent sequence of the ring $\sum_{\nu<\nu^{\prime}} \mathcal{R}_{\nu}, A_{k} \in$ $\mathcal{R}_{\nu_{k}}(k=1,2, \ldots)$, then the sequence $\xi_{\nu_{k}}\left(A_{k}\right)$ converges stochastically to some random variable. Let us put $C_{k}=\prod_{n=1}^{k} A_{n}, D_{k}=C_{k}-C_{k-1}(k=2,3, \ldots)$. As the sets $C_{1}, D_{2}, D_{3}, \ldots$ are disjoint, by applying the assumption of transfinite induction it follows that the random variables $\xi_{\nu_{1}}\left(C_{1}\right), \xi_{\nu_{2}}\left(D_{2}\right), \xi_{\nu_{3}}\left(D_{3}\right), \ldots$ are independent. Since in addition $C_{k}=C_{1}+D_{2}+\cdots+D_{k}$ and thus

$$
\xi_{\nu_{k}}\left(C_{k}\right)=\xi_{\nu_{1}}\left(C_{1}\right)+\sum_{n=2}^{k} \xi_{\nu_{n}}\left(D_{n}\right)
$$

applying the assumption of induction (3.7) we get

$$
f_{\nu_{k}}\left(t, C_{k}\right)=f_{\nu_{1}}\left(t, C_{1}\right) \prod_{n=2}^{k} f_{\nu_{n}}\left(t, D_{n}\right)=g^{*}\left(t, C_{1}\right) \prod_{n=2}^{k} g^{*}\left(t, D_{n}\right) \quad \text { if } \quad|t| \leq T .
$$

The sequence on the right hand side of (3.9) converges to a function which is continuous in the interval $[-T, T]$; consequently (see [4], p. 115, Theorem 2.7) the series on the right hand side of the expression (3.8) and thus also the sequence $\xi_{\nu_{k}}\left(C_{k}\right)$ converges with probability 1 to a limit which shall be denoted by $\xi$. We show that

$$
\xi_{\nu_{k}}\left(A_{k}\right) \Rightarrow \xi \quad \text { if } \quad k \rightarrow \infty .
$$

Consider the following relation

$$
\xi_{\nu_{k}}\left(A_{k}\right)=\xi_{\nu_{k}}\left(C_{k}\right)+\xi_{\nu_{k}}\left(A_{k}-C_{k}\right) .
$$


It suffices to show that

$$
\xi_{\nu_{k}}\left(A_{k}-C_{k}\right) \Rightarrow 0 \quad \text { if } \quad k \rightarrow \infty
$$

Since $\lim _{k \rightarrow \infty}\left(A_{k}-C_{k}\right)=0$, it follows that if $|t| \leq T$, then $\lim _{k \rightarrow \infty} f_{\nu_{k}}\left(t, A_{k}-C_{k}\right)=$ $\lim _{k \rightarrow \infty} g^{*}\left(t, A_{k}-C_{k}\right)=1$. On the other hand, by the inequality (1.3) the relation

$$
1-R f_{\nu}\left(2 t, A_{k}-C_{k}\right) \leq 4\left(1-R f\left(t, A_{k}-C_{k}\right)\right)
$$

is satisfied for every value of $t$, hence $f_{\nu_{k}}\left(t, A_{k}-C_{k}\right) \Rightarrow 1$ if $k \rightarrow \infty$; thus our statement is proved.

Let us define the set function $\xi_{\nu^{\prime}}(A)\left(A \in \mathcal{R}_{\nu^{\prime}}\right)$ as follows: if $A \in \mathcal{R}_{\nu^{\prime}}, A=\lim _{n \rightarrow \infty} A_{n}$ where $A_{n} \in \mathcal{R}_{\nu_{n}}\left(\nu_{n}<\nu^{\prime} ; n=1,2, \ldots\right)$, put

$$
\xi_{\nu^{\prime}}(A)=\operatorname{limst}_{n \rightarrow \infty} \xi_{\nu_{n}}\left(A_{n}\right)
$$

We prove that $\xi_{\nu^{\prime}}(A)$ is uniquely defined. Let $A_{1}, A_{2}, \ldots, B_{1}, B_{2}, \ldots$ be two convergent sequences of the ring $\sum_{\nu<\nu^{\prime}} \mathcal{R}_{\nu}, \lim _{n \rightarrow \infty} A_{n}=\lim _{n \rightarrow \infty} B_{n}=A$. The members of the sequences $A_{n}, B_{n}, A_{n}-B_{n}$ are also elements of the ring $\sum_{\nu<\nu^{\prime}} \mathcal{R}_{\nu}$. Besides $\lim _{n \rightarrow \infty}\left(A_{n}-\right.$ $\left.B_{n}\right)=\lim _{n \rightarrow \infty}\left(B_{n}-A_{n}\right)=0$. If $A_{n} \in \mathcal{R}_{\nu_{n}}, B_{n} \in \mathcal{R}_{\nu_{n}}\left(\nu_{n}<\nu^{\prime} ; n=1,2, \ldots\right)$, then

$$
\begin{aligned}
& \xi_{\nu_{n}}\left(A_{n}\right)=\xi_{\nu_{n}}\left(A_{n} B_{n}\right)+\xi_{\nu_{n}}\left(A_{n}-B_{n}\right), \\
& \xi_{\nu_{n}}\left(B_{n}\right)=\xi_{\nu_{n}}\left(A_{n} B_{n}\right)+\xi_{\nu_{n}}\left(B_{n}-A_{n}\right),
\end{aligned}
$$

Since $\lim _{n \rightarrow \infty}\left(A_{n}-B_{n}\right)=\lim _{n \rightarrow \infty}\left(B_{n}-A_{n}\right)=0$ and thus if $|t| \leq T$,

$$
\left.\begin{array}{l}
f_{\nu_{n}}\left(t, B_{n}-A_{n}\right)=g^{*}\left(t, B_{n}-A_{n}\right) \rightarrow 1 \\
f_{\nu_{n}}\left(t, A_{n}-B_{n}\right)=g^{*}\left(t, A_{n}-B_{n}\right) \rightarrow 1
\end{array}\right\} \text { if } n \rightarrow \infty
$$

then, taking into account the inequality (1.3), it follows that

$$
\left.\begin{array}{l}
\xi_{\nu_{n}}\left(A_{n}\right)-\xi_{\nu_{n}}\left(A_{n}, B_{n}\right) \Rightarrow 0 \\
\xi_{\nu_{n}}\left(B_{n}\right)-\xi_{\nu_{n}}\left(A_{n}, B_{n}\right) \Rightarrow 0
\end{array}\right\} \text { if } n \rightarrow \infty
$$

consequently

$$
\operatorname{limst}_{n \rightarrow \infty} \xi_{\nu_{n}}\left(A_{n}\right)=\operatorname{limst}_{n \rightarrow \infty} \xi_{\nu_{n}}\left(B_{n}\right) .
$$

Thus the definition of $\xi_{\nu^{\prime}}(A)$ is unique. This implies that

$$
\xi_{\nu^{\prime}}(A)=\xi_{\nu}(A) \quad \text { if } \quad \nu<\nu^{\prime} .
$$

Now we shall prove that all what has been supposed about the set functions $\xi_{\nu}(A)$ corresponding to the ordinal numbers $\nu<\nu^{\prime}$ will be satisfied also for the set function $\xi_{\nu^{\prime}}(A)$. First we show that $f_{\nu^{\prime}}(t, A)=g^{*}(t, A)$ if $|t| \leq T$. Put $A=\lim _{n \rightarrow \infty} A_{n}$ where 
$A_{n} \in \mathcal{R}_{\nu_{n}}\left(\nu_{n}<\nu^{\prime} ; n=1,2, \ldots\right)$. Since $\xi_{\nu_{n}}\left(A_{n}\right) \Rightarrow \xi_{\nu^{\prime}}(A)$ if $n \rightarrow \infty$ and consequently $f_{\nu_{n}}(t, A) \Rightarrow f_{\nu^{\prime}}(t, A)$, further $g^{*}\left(t, A_{n}\right) \rightarrow g^{*}(t, A)$ if $n \rightarrow \infty$, it follows that

$$
f_{\nu^{\prime}}(t, A)=\lim _{n \rightarrow \infty} f_{\nu_{n}}\left(t, A_{n}\right)=\lim _{n \rightarrow \infty} g^{*}\left(t, A_{n}\right)=g^{*}(t, A) \quad \text { if } \quad|t| \leq T .
$$

The statement that $\xi_{\nu^{\prime}}(A)$ is an additive set function can be proved as follows. Let $A_{1}, A_{2}, \ldots$ be disjoint sets belonging to the ring $\mathcal{R}_{\nu^{\prime}}$. Let $A_{1}^{(n)}, A_{2}^{(n)}, \ldots, A_{r}^{(n)}$ be sequences of sets belonging to the ring $\sum_{\nu<\nu^{\prime}} \mathcal{R}_{\nu}$ for which

$$
\lim _{n \rightarrow \infty} A_{k}^{(n)}=A_{k}, \quad k=1,2, \ldots, r, \quad A_{i}^{(n)} A_{k}^{(n)}=0 \quad \text { if } \quad i \neq k .
$$

To the sets $A_{1}^{(n)}, A_{2}^{(n)}, \ldots, A_{r}^{(n)}$ correspond independent random variables and the sum of these random variables corresponds to the sum of these sets. Since these properties hold even after carrying out the limiting process, the random variables $\xi_{\nu^{\prime}}\left(A_{1}\right), \xi_{\nu^{\prime}}\left(A_{2}\right), \ldots$, $\xi_{\nu^{\prime}}\left(A_{r}\right)$ are independent and $\xi_{\nu^{\prime}}\left(\sum_{k=1}^{r} A_{k}\right)=\sum_{k=1}^{r} \xi_{\nu^{\prime}}\left(A_{k}\right)$ is completely additive, too. In fact, as we have seen $f_{\nu^{\prime}}(t, A)=g^{*}(t, A)$ if $|t| \leq T$ and for every non-increasing sequence $A_{1}, A_{2}, \ldots$ of sets belonging to $\mathcal{R}_{\nu^{\prime}}$ for which $\lim _{n \rightarrow \infty} A_{n}=0$, we have

$$
\lim _{n \rightarrow \infty} f_{\nu^{\prime}}\left(t, A_{n}\right)=\lim _{n \rightarrow \infty} g^{*}\left(t, A_{n}\right)=1 \quad(|t| \leq T) .
$$

Thus the condition of the Corollary of Theorem 2.1 is satisfied, hence our assertion holds.

Finally, let us define the set function $\xi^{*}(A)$ as follows:

$$
\xi^{*}(A)=\xi_{\nu}(A) \quad \text { if } \quad A \in \mathcal{R}_{\nu} \quad\left(\nu<\omega_{1}\right) .
$$

Since $\sum_{\nu} \mathcal{R}_{\nu}=\mathcal{S}(\mathcal{R})$, the set function $\xi^{*}(A)$ has been defined for every element $A$ of $\mathcal{S}(\mathcal{R})$. It is clear that $\xi^{*}(A)$ is an additive set function. $\xi^{*}(A)$ is even completely additive. This follows from the fact that, as we have seen, for the characteristic functions $f^{*}(t, A)$ of the random variables $\xi^{*}(A)$ the relation $f^{*}(t, A)=g^{*}(t, A)(|t| \leq T)$ holds. Thus for every non-increasing sequence of $\mathcal{S}(\mathcal{R})$ tending to 0 , we have

$$
\lim _{n \rightarrow \infty} f^{*}\left(t, A_{n}\right)=\lim _{n \rightarrow \infty} g^{*}\left(t, A_{n}\right)=1 \quad \text { if } \quad|t| \leq T
$$

which according to the Corollary of Theorem 2.1 implies that $\xi^{*}(A)$ is a completely additive set function.

The uniqueness of the extension can be proved as follows. Let $\xi^{*}$ and $\xi^{* *}$ be two completely additive set functions defined on the $\sigma$-ring $\mathcal{S}(\mathcal{R})$. Suppose that $\xi^{*}$ and $\xi^{* *}$ coincide on the ring $\mathcal{R}$. Let $\mathfrak{M}$ denote the class of those sets $A$ for which $\xi^{*}(A)=$ $\xi^{* *}(A)$. Let $A_{1}, A_{2}, \ldots$ be a monotone sequence of sets $\mathfrak{M}, A=\lim _{n \rightarrow \infty} A_{n}$. The complete additiveness of the set functions $\xi^{*}, \xi^{* *}$ implies that

$$
\lim _{n \rightarrow \infty} \xi^{*}\left(A_{n}\right)=\xi^{*}(A), \quad \lim _{n \rightarrow \infty} \xi^{* *}\left(A_{n}\right)=\xi^{* *}(A) .
$$


Since $\xi^{*}\left(A_{n}\right)=\xi^{* *}\left(A_{n}\right)(n=1,2, \ldots)$, it follows that $A \in \mathfrak{M}$. Hence $\mathfrak{M}$ is a monotone class of sets. We know that $\mathcal{R} \subseteq \mathfrak{M}, \mathfrak{M} \subseteq \mathcal{S}(\mathcal{R})$ but these together imply that $\mathfrak{M}=\mathcal{S}(\mathcal{R})$, because the smallest monotone class containing a ring $\mathcal{R}$ is identical with the $\sigma$-ring $\mathcal{S}(\mathcal{R})$.

PROOF OF THE SECOND PART OF THE THEOREM. In order to prove the second half of the theorem we shall show that for any positive number $T$ the conditions of Theorem 1.1 are satisfied for the set function (3.5). Condition a) is obviously satisfied because $f(t, A)$ is a characteristic function and accordingly

$$
\sup _{|t| \leq T}|1-f(t, A)| \leq 2
$$

Let us investigate Condition b). By Theorem 2.7 the set function $|1-f(t, A)|$ is subadditive for every fixed value of $t$. Hence it follows that if $T$ is a fixed positive number, then the set function $\sup _{|t| \leq T}|1-f(t, A)|$ is also subadditive, i.e. Condition $\mathrm{b}$ ) is also satisfied.

Finally, as for Condition c), let us consider an arbitrary sequence of disjoint sets $A_{1}, A_{2}, \ldots$ of the $\sigma$-ring $\mathcal{S}$. Since the series

$$
\sum_{k=1}^{\infty} \xi\left(A_{k}\right)
$$

converges with probability 1 regardless of the order of summation, it follows that the infinite series

$$
\sum_{k=1}^{\infty} \int_{|x| \leq 1} x^{2} \mathrm{~d} F\left(x, A_{k}\right), \quad \sum_{k=1}^{\infty}\left|\int_{|x| \leq 1} x \mathrm{~d} F\left(x, A_{k}\right)\right|, \quad \sum_{k=1}^{\infty} \mathbf{P}\left(\left|\xi\left(A_{k}\right)\right|>1\right)
$$

are convergent $([8], \S 5)$. Hence by the inequality

$$
\begin{aligned}
\left|1-f\left(t, A_{k}\right)\right|= & \left|\int_{-\infty}^{\infty}\left(1-e^{i t x}\right) \mathrm{d} F\left(x, A_{k}\right)\right|=\left|\int_{|x| \leq 1}+\int_{|x|>1}\right| \\
\leq & \left|\int_{|x| \leq 1}\left(e^{i t x}-1-i t x\right) \mathrm{d} F\left(x, A_{k}\right)\right|+|t|\left|\int_{|x| \leq 1} x \mathrm{~d} F\left(x, A_{k}\right)\right| \\
& +2 \mathbf{P}\left(\left|\xi\left(A_{k}\right)\right|>1\right) \leq \frac{t^{2}}{2} \int_{|x| \leq 1} x^{2} \mathrm{~d} F\left(x, A_{k}\right) \\
& +|t|\left|\int_{|x| \leq 1} x \mathrm{~d} F\left(x, A_{k}\right)\right|+2 \mathbf{P}\left(\left|\xi\left(A_{k}\right)\right|>1\right)
\end{aligned}
$$

it follows that Condition c) is also satisfied, i.e. the set function (3.6) is of bounded variation. Thus Theorem 3.1 is proved.

As a consequence of Theorem 3.1 we can prove 
TheOREm 3.2. Let $\xi(A)$ be a completely additive set function defined on a ring $\mathcal{R}$. If for every sequence $A_{1}, A_{2}, \ldots$ of disjoint sets of $\mathcal{R}$ the series

$$
\sum_{k=1}^{\infty} \xi\left(A_{k}\right)
$$

converges with probability 1 , then $\xi(A)$ can be extended to $\mathcal{S}(\mathcal{R})$.

Proof. The above series must converge with probability 1 regardless of the order of summation, since a rearrangement $A_{i_{k}}$ of the sequence $A_{k}$ consists also of disjoint sets of $\mathcal{R}$. Hence by Theorem 2.1 of [4] (p. 115) it follows that

$$
\sum_{k=1}^{\infty}\left|1-f\left(t, A_{k}\right)\right|<\infty
$$

for every $t$. By Theorems 2.7 and 1.1, for every $t,|1-f(t, A)|(A \in \mathcal{R})$ is of bounded variation, and thus the conditions in Theorem 3.1 are fulfilled. This completes the proof of the theorem.

The proof of the following theorem is contained implicitly in the proof of Theorem 3.1. However, we give here another proof which does not use transfinite induction and is based only on the second assertion of Theorem 3.1.

THEOREM 3.3. Let $\xi(A)$ be a completely additive set function defined on a $\sigma$-ring $\mathcal{S}$. If $A_{1}, A_{2}, \ldots$ is a convergent sequence of sets of $\mathcal{S}, \lim _{n \rightarrow \infty} A_{n}=A$, then

$$
\xi\left(A_{n}\right) \Rightarrow \xi(A) \quad \text { if } \quad n \rightarrow \infty .
$$

Proof. By Theorem 2.7, for every fixed $t,|1-f(t, A)|$ is a completely additive set function. Hence it follows that for every $T>0, \sup _{t \leq T}|1-f(t, A)|$ is also completely subadditive and by Theorem 3.1 it is also of bounded variation. Let $W(T, A)$ denote the variation of $\sup _{|t| \leq T}|1-f(t, A)|$ on the set $A \in \mathcal{S}$, then by Theorem 1.2 it follows that $W(T, A)$ is a bounded measure on $\mathcal{S}$. Since $\lim _{n \rightarrow \infty}\left(A_{n}-A\right)=\lim _{n \rightarrow \infty}\left(A-A_{n}\right)=0$, we have $\lim _{n \rightarrow \infty} W\left(T, A_{n}-A\right)=\lim _{n \rightarrow \infty} W\left(T, A-A_{n}\right)=0$. Taking into account the inequality

$$
\sup _{|t| \leq T}|1-f(t, B)| \leq W(T, B) \quad(B \in \mathcal{S})
$$

we obtain that $\lim _{n \rightarrow \infty} f\left(t, A_{n}-A\right)=\lim _{n \rightarrow \infty} f\left(t, A-A_{n}\right)=1$ if $|t| \leq T$. As this is true for every $T>0$, it follows that

$$
\xi\left(A-A_{n}\right) \Rightarrow 0, \quad \xi\left(A_{n}-A\right) \Rightarrow 0 \quad \text { if } \quad n \rightarrow \infty .
$$

From this fact and from the inequality

$$
\left|\xi\left(A_{n}\right)-\xi(A)\right| \leq\left|\xi\left(A_{n}-A\right)\right|+\left|\xi\left(A-A_{n}\right)\right|
$$

our assertion follows immediately. 


\section{$\S 3$. An extension theorem under condition on the distribu- tion functions}

In the following theorems the possibility of carrying out the extension will be proved by reducing it to Theorem 3.1. Thus we need not state again the uniqueness of the extension.

THEOREM 3.4. Let $\xi(A)$ be a completely additive set function defined on a ring $\mathcal{R}$. If the set of distribution functions $\{F(x, A), A \in \mathcal{R}\}$ is compact, then $\xi(A)$ can be extended to $\mathcal{S}(\mathcal{R})$.

Conversely, if $\xi(A)$ is a completely additive set function defined on a $\sigma$-ring $\mathcal{S}$, then the set $\{F(x, A), A \in \mathcal{S}\}$ is compact.

Proof of the first part of the theorem. Let $A_{1}, A_{2}, \ldots$ be a sequence of disjoint sets of $\mathcal{R}$. The set $\mathcal{F}^{\prime}=\left\{F\left(x, A_{i_{1}}+A_{i_{2}}+\cdots+A_{i_{r}}\right)\right\}$ is a subset of $\{F(x, A), A \in \mathcal{R}\}$, hence $\mathcal{F}^{\prime}$ is compact. According to Theorem 1.10, the series

$$
\sum_{k=1}^{\infty} \xi\left(A_{k}\right)
$$

converges with probability 1 regardless of the order of summation, hence by Theorem 3.2 $\xi(A)$ can be extended to $\mathcal{S}(\mathcal{R})$.

Proof of THE SECOND PART OF THE THEOREM. First we prove that the set function $\mu_{3}(A)(\in \mathcal{S})$ is completely subadditive. Let $A_{1}, A_{2}, \ldots$ be a sequence of disjoint sets of the $\sigma$-ring $\mathcal{S}$. By Theorem 2.5 it follows that

$$
\mu_{3}\left(\sum_{k=1}^{\infty} A_{k}\right) \leq \sum_{k=1}^{n} \mu_{3}\left(A_{k}\right)+\mu_{3}\left(\sum_{k=n+1}^{\infty} A_{k}\right) .
$$

Consequently, we have merely to show that the second member on the right hand side converges to 0 . Let $B_{n}=\sum_{k=n}^{\infty} A_{k}$. The set function $\mu_{3}(A)$ is monotonous, hence the sequence $\mu_{3}\left(B_{n}\right)$ is non-increasing. By Theorem $2.6 \mu_{3}(A)$ can take on only the values 0 and 1, therefore there are two cases: either there is an $N$ such that $\mu_{3}\left(B_{n}\right)=0$ if $n>N$ or $\mu_{3}\left(B_{n}\right)=1$ for all values of $n$. Contrarily to the statement let us suppose that the latter case holds. Then by the Corollary of Theorem 1.4 and by Theorem 2.2 there exists a sequence $C_{1}, C_{2}, \ldots$ of sets such that $C_{k} \in \mathcal{S}, C_{k} \subseteq B_{k},\left|Q\left(\frac{1}{2}, C_{k}\right)\right|>1(k=1,2, \ldots)$. Since $B_{k} \rightarrow 0$, it follows that $C_{k} \rightarrow 0$, hence by Theorem $3.2 \xi\left(C_{k}\right) \Rightarrow 0$ if $k \rightarrow \infty$. Thus $Q\left(\frac{1}{2}, C_{k}\right) \rightarrow 0$ if $k \rightarrow \infty$, what is a contradiction. Consequently, $\mu_{3}(A)$ is completely subadditive. 
It follows from the monotonity of the set function $\mu_{3}(A)$ that the following property also holds: if $A_{1}, A_{2}, \ldots$ are arbitrary sets belonging to the $\sigma$-ring $\mathcal{S}$, then

$$
\mu_{3}\left(\sum_{k=1}^{\infty} A_{k}\right) \leq \sum_{k=1}^{\infty} \mu_{3}\left(A_{k}\right) .
$$

Namely

$$
\sum_{k=1}^{\infty} A_{k}=\sum_{k=1}^{\infty}\left(A_{k}-\sum_{l=1}^{k-1} A_{l}\right) \quad\left(A_{0}=0\right),
$$

therefore, since $\mu_{3}(A)$ is completely subadditive and monotonically increasing, it follows that

$$
\mu_{3}\left(\sum_{k=1}^{\infty} A_{k}\right) \leq \sum_{k=1}^{\infty} \mu_{3}\left(A_{k}-\sum_{l=1}^{k-1} A_{l}\right) \leq \sum_{k=1}^{\infty} \mu_{3}\left(A_{k}\right) .
$$

Consider the completely additive set function $\xi(A)$ defined on the $\sigma$-ring $\mathcal{S}$. In contradiction to the statement, let us suppose that the set $\{F(x, A), A \in \mathcal{S}\}$ is not compact. Then, by Theorem 1.6,

$$
\mu_{3}=\lim _{\varepsilon \rightarrow \infty} \sup _{A \in \mathcal{S}} \mathbf{P}(|\xi(A)|>\varepsilon)=\rho>0
$$

Hence it follows that for every positive $\varepsilon$

$$
\sup _{A \in \mathcal{S}} \mathbf{P}(|\xi(A)|>\varepsilon) \geq \rho>0 .
$$

Since

$$
\sup _{A \in \mathcal{S}} \mathbf{P}(|\xi(A)|>\varepsilon)=\sup _{A \in \mathcal{S}} \sup _{B \in A \mathcal{S}} \mathbf{P}(|\xi(B)|>\varepsilon),
$$

it follows that there exists a sequence of sets $A_{n}\left(A_{n} \in \mathcal{S}\right)$ for which

$$
\sup _{B \in A_{n} \mathcal{S}} \mathbf{P}\left(\left|\xi\left(B_{n}\right)\right|>n\right) \geq \frac{\rho}{2}>0
$$

If $A=\sum_{k=1}^{\infty} A_{k}$, then by the preceding inequalities

$$
\sup _{B \in A \mathcal{S}} \mathbf{P}(|\xi(B)|>n) \geq \sup _{B \in A_{n} \mathcal{S}} \mathbf{P}(|\xi(B)|>n) \geq \frac{\rho}{2}>0 ;
$$

therefore

$$
\mu_{3}(A)=\lim _{n \rightarrow \infty} \sup _{B \in A \mathcal{S}} \mathbf{P}(|\xi(B)|>n) \geq \frac{\rho}{2}>0 .
$$

By Theorem $2.6 \mu_{3}(A)$ can take on only the values 0 and 1 ; hence it follows that $\mu_{3}(A)=1$. 
Now we shall prove that if $B \in \mathcal{S}, \mu_{3}(B)=1$, then for every pair of numbers $\lambda, m$ we can found a set $B_{m} \in B \mathcal{S}$ such that by a convenient choice of the quantiles $Q\left(\lambda, B_{m}\right)$ we have

$$
\mu_{3}\left(B_{m}\right)=1, \quad\left|Q\left(\lambda, B_{m}\right)\right|>m .
$$

Suppose that such a set does not exist. Since $\mu_{3}(B)=1$, we can find sets $C_{k} \in B \mathcal{S}$ $(k=1,2, \ldots)$ such that $\left|Q\left(\lambda, C_{k}\right)\right|>k$. According to our assumption $\mu_{3}\left(C_{k}\right)=0$ if $k \geq m$. Consider the set $C=\sum_{k=m}^{\infty} C_{k}$. We have proved that

$$
\mu_{3}(C) \leq \sum_{k=m}^{\infty} \mu_{3}\left(C_{k}\right)
$$

therefore $\mu_{3}(C)=0$. Then by Theorems 1.6 and 1.5 , there exists a number $K(\lambda)$ such that for all $Q\left(\lambda, C^{\prime}\right)$ we have

$$
\left|Q\left(\lambda, C^{\prime}\right)\right| \leq K(\lambda) \quad \text { if } \quad C^{\prime} \in C \mathcal{S}
$$

but this is a contradiction.

Applying to the set $B=A$ what has been said above let us choose a set $B_{1} \in A S$ for which $\mu_{3}\left(B_{1}\right)=1,\left|Q\left(\frac{1}{2}, B_{1}\right)\right|>1$. Similarly, it follows that there exists a set $B_{2} \in B_{1} \mathcal{S}$ for which $\mu_{3}\left(B_{2}\right)=1,\left|Q\left(\frac{1}{2}, B_{2}\right)\right|>2$ etc. Therefore we can construct a non-increasing sequence of sets $B_{1}, B_{2}, \ldots$ for which $B_{n} \in \mathcal{S},\left|Q\left(\frac{1}{2}, B_{n}\right)\right|>n$. The sequence $\xi\left(B_{n}\right)$ is convergent because

$$
\xi\left(B_{n}\right)=\xi(B)+\sum_{k=n}^{\infty}\left(\xi\left(B_{k}\right)-\xi\left(B_{k+1}\right)\right), \quad B=\prod_{k=1}^{\infty} B_{k} .
$$

Consequently, the series $Q\left(\frac{1}{2}, B_{n}\right)$ is bounded, but this is a contradiction. Thus Theorem 3.2 is proved.

Corollary. Let $\xi(A)$ be a completely additive set function defined on a ring $\mathcal{R}$. If

$$
\mu_{3}=\lim _{\varepsilon \rightarrow \infty} \sup _{A \in \mathcal{R}} \mathbf{P}(|\xi(A)|>\varepsilon)=0,
$$

then $\xi(A)$ can be extended to $\mathcal{S}(\mathcal{R})$.

Conversely, if $\xi(A)$ is a completely additive set function defined on a $\sigma$-ring $\mathcal{S}$, then

$$
\mu_{3}=\lim _{\varepsilon \rightarrow \infty} \sup _{A \in \mathcal{S}} \mathbf{P}(|\xi(A)|>\varepsilon)=0 .
$$

Proof. The statement follows from Theorems 3.4 and 1.6 immediately. 


\section{$\S 4$. Extension theorems under conditions on quantiles}

The following theorems are simple applications of Theorems 3.1 and 3.2 and of those of Chapter II and deal with the reduction of the condition in Theorem 3.4.

Theorem 3.4 contains when compared with the case of ordinary real-valued set functions a surplus of conditions which are needed to ensure the extension of a completely additive random-valued set function $\xi(A)$. If $\varphi(A)$ is a completely additive real-valued set function defined on a ring $\mathcal{R}$ and

$$
\xi(\omega, A) \equiv \varphi(A) \quad \text { if } \quad A \in \mathcal{R},
$$

then

$$
Q(\lambda, A) \equiv \varphi(A) \quad \text { if } \quad 0<\lambda<1, \quad A \in \mathcal{R},
$$

i.e. all quantiles of $\xi(\omega, A)$ coincide with the value $\varphi(A)$. Since it has been shown that for the extension of $\varphi(A)$ the boundedness of the set $\{\varphi(A), A \in \mathcal{R}\}$ is needed, we can say that the set function $\xi(A)=\varphi(A)$ can be extended if there is a $\lambda$ for which the set $\{Q(\lambda, A), A \in \mathcal{R}\}$ is bounded.

If the random variables $\xi(A)(A \in \mathcal{R})$ are not constants, then for the extension the boundedness of two quantile-sets is required.

THEOREM 3.5. Let $\xi(A)$ be a completely additive set function defined on a ring $\mathcal{R}$. If there exists a pair of numbers $\lambda_{1}, \lambda_{2}\left(0<\lambda_{1}<\lambda_{2}<1\right)$ such that with a convenient choice of the quantiles $Q\left(\lambda_{1}, A\right), Q\left(\lambda_{2}, A\right)$ the sets $\left\{Q\left(\lambda_{1}, A\right), A \in \mathcal{R}\right\},\left\{Q\left(\lambda_{2}, A\right), A \in \mathcal{R}\right\}$ are bounded, then $\xi(A)$ can be extended to $\mathcal{S}(\mathcal{R})$.

Proof. The theorem is a straightforward consequence of Theorems 1.11 and 3.2.

THEOREM 3.6. Let $\xi(A)$ be a completely additive set function defined on a ring $\mathcal{R}$. If the random variables $\xi(A)(A \in \mathcal{R})$ are symmetrically distributed and there exists a number $\lambda \neq \frac{1}{2}(0<\lambda<1)$ such that with a convenient choice of the quantiles $Q(\lambda, A)$ the set $\{Q(\lambda, A), A \in \mathcal{R}\}$ is bounded, then $\xi(A)$ can be extended to $\mathcal{S}(\mathcal{R})$.

Proof. Since $\lambda \neq \frac{1}{2}$, it follows that $\lambda \neq 1-\lambda$. On the other hand, $\xi(A)$ has a symmetric distribution, hence with a convenient choice of the quantiles $Q(1-\lambda, A)$ the set $\{Q(1-\lambda, A), A \in \mathcal{R}\}$ is also bounded together with the set $\{Q(\lambda, A), A \in \mathcal{R}\}$ and thus the conditions of Theorem 3.5 are satisfied.

TheOREm 3.7. Let $\xi(A)$ be a completely additive set function defined on a ring $\mathcal{R}$. If the random variables $\xi(A)$ are non-negative and there is a $\lambda(0<\lambda<1)$ such that with a convenient choice of the quantiles $Q(\lambda, A)$ the set $\{Q(\lambda, A), A \in \mathcal{R}\}$ is bounded, then $\xi(A)$ can be extended to $\mathcal{S}(\mathcal{R})$.

PROOF. The theorem is a straightforward consequence of Corollary 2 of Theorem 1.11 and of Theorem 3.2 . 


\section{$\S 5$. Further extension theorems}

TheOREm 3.8. ${ }^{6}$ Let $\xi(A)$ be a completely additive set function defined on a ring $\mathcal{R}$. If there is a positive number $\varepsilon$ such that the following set functions

$$
\int_{|x| \leq \varepsilon} x^{2} \mathrm{~d} F(x, A), \quad \int_{|x| \leq \varepsilon} x \mathrm{~d} F(x, A), \quad \mathbf{P}(|\xi(A)|>\varepsilon) \quad(A \in \mathcal{R})
$$

are of bounded variation, then $\xi(A)$ can be extended to $\mathcal{S}(\mathcal{R})$.

Conversely, if $\xi(A)$ is a completely additive set function defined on a $\sigma$-ring $\mathcal{S}$, then the set functions (3.10) $(A \in \mathcal{S})$ are of bounded variation for every positive $\varepsilon$.

Proof OF THE FIRST PART OF THE THEOREM. We shall show that the set function $|1-f(t, A)|$ is of bounded variation for every fixed value of $t$. Namely, if $\varepsilon>0$, then

$$
\begin{gathered}
|1-f(t, A)|=\left|\int_{-\infty}^{\infty}\left(1-e^{i t x}\right) \mathrm{d} F(x, A)\right| \\
\leq \frac{t^{2}}{2} \int_{|x| \leq \varepsilon} x^{2} \mathrm{~d} F(x, A)+|t|\left|\int_{|x| \leq \varepsilon} x \mathrm{~d} F(x, A)\right|+2 \mathbf{P}(|\xi(A)|>\varepsilon) \quad(A \in \mathcal{R}) .
\end{gathered}
$$

Proof of the SECOND PART OF THE TheOREM. By Theorem 3.1 the set function (3.5) is of bounded variation. By inequality (1.1) it follows that if $0<\varepsilon \leq 1$, the set function

$$
\int_{|x| \leq \varepsilon} x^{2} \mathrm{~d} F(x, A)
$$

is of bounded variation.

By inequality (1.2) it follows that

$$
\mathbf{P}(|\xi(A)|>\varepsilon) \quad(A \in \mathcal{S})
$$

is of bounded variation for every $\varepsilon>0$. In addition, since for an arbitrary positive $\varepsilon$ we have

$$
\int_{|x| \leq \varepsilon} x^{2} \mathrm{~d} F(x, A) \leq \int_{|x| \leq 1} x^{2} \mathrm{~d} F(x, A)+\varepsilon^{2} \mathbf{P}(|\xi(A)|>1),
$$

taking into account what has been said previously we obtain that the set function

$$
\int_{|x| \leq \varepsilon} x^{2} \mathrm{~d} F(x, A)
$$

\footnotetext{
${ }^{6}$ This theorem can be regarded as a generalization of the three series theorem of KoLMOGOROV.
} 
is also of bounded variation for every positive $\varepsilon$. Let us consider the following inequality:

$$
\begin{aligned}
f(t, A)-1= & \int_{|x| \leq \varepsilon}\left(e^{i t x}-1-i t x\right) \mathrm{d} F(x, A) \\
& +i t \int_{|x| \leq \varepsilon} x \mathrm{~d} F(x, A)+\int_{|x|>\varepsilon}\left(e^{i t x}-1\right) \mathrm{d} F(x, A) .
\end{aligned}
$$

If $t \neq 0$, it follows

$$
\left|\int_{|x| \leq \varepsilon} x \mathrm{~d} F(x, A)\right| \leq \frac{1}{|t|}|1-f(t, A)|+\frac{|t|}{2} \int_{|x| \leq \varepsilon} x^{2} \mathrm{~d} F(x, A)+\frac{2}{|t|} \mathbf{P}(|\xi(A)|>\varepsilon),
$$

hence the set functions (3.10) are of bounded variation for every positive $\varepsilon$. Thus we have proved the theorem.

THEOREM 3.9. Let $\xi(A)$ be a completely additive set function defined on a ring $\mathcal{R}$. If the set function

$$
1-P_{0}(A)=1-\mathbf{P}(\xi(A)=0)
$$

is of bounded variation, then $\xi(A)$ can be extended to $\mathcal{S}(\mathcal{R})$.

Proof. The theorem is a straightforward consequence of the inequality

$$
|1-f(t, A)| \leq 2\left(1-P_{0}(A)\right)
$$

and of Theorem 3.1.

THEOREM 3.10. Let $\xi(A)$ be a completely additive set function defined on a ring $\mathcal{R}$. If the set function

$$
\mathbf{M}(|\xi(A)|)=\int_{-\infty}^{\infty}|x| \mathrm{d} F(x, A) \quad(A \in \mathcal{R})
$$

is of bounded variation, then $\xi(A)$ can be extended to $\mathcal{S}(\mathcal{R})$.

Proof. The theorem is an immediate consequence of the inequality

$$
|1-f(t, A)|=\left|\int_{-\infty}^{\infty}\left(1-e^{i t x}\right) \mathrm{d} F(x, A)\right| \leq|t| \int_{-\infty}^{\infty}|x| \mathrm{d} F(x, A)
$$

and of Theorem 3.1.

THEOREM 3.11. Let $\xi(A)$ be a completely additive set function defined on a ring $\mathcal{R}$. If the set functions

$$
\begin{gathered}
M(A)=\mathbf{M}(\xi(A))=\int_{-\infty}^{\infty} x \mathrm{~d} F(x, A), \\
D^{2}(A)=\mathbf{D}^{2}(\xi(A))=\int_{-\infty}^{\infty} x^{2} \mathrm{~d} F(x, A)-\left(\int_{-\infty}^{\infty} x \mathrm{~d} F(x, A)\right)^{2}
\end{gathered}
$$

are of bounded variation, then $\xi(A)$ can be extended to $\mathcal{S}(\mathcal{R})$. 
Proof. According to Chebysev's inequality

$$
\mathbf{P}(|\xi(A)-M(A)|>\varepsilon) \leq \frac{D^{2}(A)}{\varepsilon^{2}} .
$$

Consequently, if $|M(A)| \leq M_{1}, D^{2}(A) \leq D_{1}$, then

$$
\mathbf{P}\left(|\xi(A)|>M_{1}+\varepsilon\right) \leq \mathbf{P}(|\xi(A)-M(A)|>\varepsilon) \leq \frac{D^{2}(A)}{\varepsilon^{2}} \leq \frac{D_{1}}{\varepsilon^{2}} .
$$

Hence we have

$$
\mu_{3}=\lim _{\varepsilon \rightarrow \infty} \sup _{A \in \mathcal{R}} \mathbf{P}\left(|\xi(A)|>M_{1}+\varepsilon\right)=0,
$$

therefore, by Theorem 1.6 and the Corollary of Theorem 3.4, the extension can be carried out.

REMARK. We see that the set functions $M(A)$ and $D^{2}(A)$ need not to be completely additive. The extension can be carried out also if they are only bounded.

\section{$\S$ 6. Extension of a set function defined on an algebra}

If the domain of definition of the set function $\xi(A)$ is an algebra, the conditions concerning the extension can be reduced. This is shown by the following theorems:

THEOREM 3.12. Let $\xi(A)$ be a completely additive set function defined on an algebra $\mathcal{R}$. If there exists a number $\lambda(0<\lambda<1)$ such that by a convenient choice of the quantiles $Q(\lambda, A)$ the set $\{Q(\lambda, A), A \in \mathcal{R}\}$ is bounded, then $\xi(A)$ can be extended to $\mathcal{S}(\mathcal{R})$.

Proof. The theorem is a straightforward consequence of Theorems 2.2, 1.5 and 3.2.

TheOREM 3.13. Let $\xi(A)$ be a completely additive set function defined on an algebra $\mathcal{R}$. If the random variables $\xi(A)(A \in \mathcal{R})$ are symmetrically distributed, then the set function $\xi(A)$ can be extended to $\mathcal{S}(\mathcal{R})$.

Proof. Since the quantiles $Q\left(\frac{1}{2}, A\right)$ can be chosen in such a way that $Q\left(\frac{1}{2}, A\right)=0$, the statement follows from Theorem 3.12 immediately.

TheOrem 3.14. Let $\xi(A)$ be a completely additive set function defined on an algebra $\mathcal{R}$. If $\xi(A) \geq 0(A \in \mathcal{R})$, then the set function $\xi(A)$ can be extended to $\mathcal{S}(\mathcal{R})$. 
Proof. From

$$
\xi(H)=\xi(A)+\xi(\bar{A}) \quad(A \in \mathcal{R})
$$

it follows that $\xi(A) \leq \xi(H)$. Hence if $Q(\lambda, H)$ is the greatest $\lambda$-quantile of $\xi(H)$, then

$$
0 \leq Q(\lambda, A) \leq Q(\lambda, H)=K(\lambda),
$$

therefore by Theorems 1.5 and $3.4 \xi(A)$ can be extended to $\mathcal{S}(\mathcal{R})$.

\section{$\S 7$. The case of Euclidean spaces}

There often occur problems in which we need to have an additive set function $\xi(A)$ with given properties on the ring of the bounded Borel sets of the space $\mathcal{R}_{n}$. In this case we act in such a way that we divide the space $R_{n}$ into a sum of an enumerable number of $n$-dimensional intervals in each of which the extension can be carried out.

In the present case $H$ is the $n$-dimensional Euclidean space, $H=\mathcal{R}_{n}$ and $\mathcal{R}$ is the ring whose elements are finite sums of $n$-dimensional intervals of the following type:

$$
a_{k} \leq x_{k}<b_{k} \quad(k=1,2, \ldots, n) .
$$

Let $\xi(A)$ be a completely additive set function defined on the ring $\mathcal{R}$. Suppose that $H$ can be divided into a countable sum of disjoint intervals $H_{1}, H_{2}, \ldots$ which have the property that every bounded set can be covered with a finite number of the intervals $H_{k}$ and $\xi(A)$ can be extended to the $\sigma$-algebras $H_{k} \mathcal{R}$. Under these conditions there is a set function $\xi^{*}(B)$ is completely additive and

$$
\xi^{*}(B)=\xi(B) \quad \text { if } \quad B \in \mathcal{R} .
$$

The extension is unique, i.e. if $\xi^{* *}(B)$ is a completely additive set function defined on the ring $\mathcal{B}_{1}$ and

$$
\xi^{*}(B)=\xi^{* *}(B) \quad \text { if } \quad B \in \mathcal{R},
$$

then

$$
\xi^{*}(B)=\xi^{* *}(B) \quad \text { if } \quad B \in \mathcal{B}_{1}
$$

This can be seen as follows. Carry out the extension inside the sets $H_{k}$. If $B \in \mathcal{B}_{1}$, then there is a number $N$ such that $B \subseteq \sum_{k=1}^{N} H_{k}$. Let

$$
\xi^{*}(B)=\sum_{k=1}^{N} \xi^{*}\left(B H_{k}\right) .
$$

It is easy to see that $\xi^{*}(B)$ is completely additive on the ring $\mathcal{B}_{1}$ and on the elements of $\mathcal{R}$ it coincides with $\xi(B)$. Also the statement concerning the uniqueness can be simply proved. 
From the precedings and from Theorems 3.11 and 3.12 it follows immediately that if $\mathcal{R}, \mathcal{B}_{1}$ and $\xi(A)$ denote the same as above and for every set $A \in \mathcal{R}$ the variable $\xi(A)$ has a symmetrical distribution or for every set $A \in \mathcal{R}$ we have $\xi(A) \geq 0$, then the set function $\xi(A)$ can be extended to the ring $\mathcal{B}_{1}$.

In the following theorem we consider as random variables also the functions $\xi(\omega), \omega \in \Omega$ which are measurable but eventually may have an infinite value with positive probability.

TheOREM 3.15. Denote by $\mathcal{R}$ the same ring as above. Let $\mathcal{R}$ be the $\sigma$-algebra of Borel sets of the space $R_{n}$ and $\xi(A)$ a completely additive set function defined on the elements of $\mathcal{R}$ for which

$$
0 \leq \xi(A)<\infty \quad(A \in \mathcal{R}) .
$$

Under these conditions $\xi(A)$ can be extended to all elements of $\mathcal{B}$ and the extension is unique.

Proof. First, according to what has been said above, let us carry out the extension of $\xi(A)$ to the ring $\mathcal{B}_{1}$. Now, if $B$ is an unbounded Borel set and $B=\sum_{k=1}^{\infty} B_{k}$ where the sets $B_{1}, B_{2}, \ldots$ are bounded disjoint Borel sets, then put

$$
\xi^{*}(B)=\sum_{k=1}^{\infty} \xi^{*}\left(B_{k}\right)
$$

This correspondence is unique. In fact, if $C_{1}, C_{2}, \ldots$ is a sequence of bounded disjoint Borel sets for which $B=\sum_{k=1}^{\infty} C_{k}$, then, according to

$$
B_{n}=\sum_{k=1}^{\infty} B_{n} C_{k}
$$

and

$$
C_{k}=\sum_{n=1}^{\infty} C_{k} B_{n}
$$

it follows that

$$
\xi^{*}\left(B_{n}\right)=\sum_{k=1}^{\infty} \xi^{*}\left(B_{n} C_{k}\right), \quad \xi^{*}\left(C_{k}\right)=\sum_{n=1}^{\infty} \xi^{*}\left(C_{k} B_{n}\right)
$$

Thus

$$
\sum_{n=1}^{\infty} \xi^{*}\left(B_{n}\right)=\sum_{n=1}^{\infty} \sum_{k=1}^{\infty} \xi^{*}\left(B_{n} C_{k}\right)=\sum_{k=1}^{\infty} \sum_{n=1}^{\infty} \xi^{*}\left(C_{k} B_{n}\right)=\sum_{k=1}^{\infty} \xi^{*}\left(C_{k}\right)
$$

$\xi^{*}(B)$ is a completely additive set function. Namely, by the construction it is clear that if the sets $B_{1}, B_{2}, \ldots, B_{r}$ are pairwise disjoint sets, then the random variables $\xi^{*}\left(B_{1}\right)$, $\xi^{*}\left(B_{2}\right), \ldots, \xi^{*}\left(B_{r}\right)$ are independent. If $B=\sum_{k=1}^{r} B_{k}$, where $B_{k}$ is a sequence of disjoint 
Borel sets, let us construct the sequences $\left\{C_{k n}\right\}$ consisting of bounded Borel sets for which we have

$$
B_{k}=\sum_{n=1}^{\infty} C_{k n}, \quad C_{k n} C_{k m}=0 \quad \text { if } \quad n \neq m \quad(k=1,2, \ldots) .
$$

We know that

$$
\xi^{*}\left(B_{k}\right)=\sum_{n=1}^{\infty} \xi^{*}\left(C_{k n}\right),
$$

therefore, by the uniqueness of the definition of $\xi^{*}(B)$, it follows that,

$$
\xi^{*}(B)=\sum_{k, n} \xi^{*}\left(C_{k n}\right)=\sum_{k=1}^{\infty} \sum_{n=1}^{\infty} \xi^{*}\left(C_{k n}\right)=\sum_{k=1}^{\infty} \xi^{*}\left(B_{k}\right) .
$$

This completes the proof of the theorem.

REMARK 1. The random variables $\xi^{*}(B)(B \in \mathcal{B})$ are either finite-valued with probability 1 or infinite-valued with probability 1 . Namely, if $B=\sum_{k=1}^{\infty} B_{k}$ where $B_{i} B_{k}=0$ if $i \neq k, B_{k} \in \mathcal{B}_{1}(k=1,2, \ldots)$, then the probability that the sum of the series $\sum_{k=1}^{\infty} \xi^{*}\left(B_{k}\right)$ is finite is either 0 or 1 ([8], p. 60).

REMARK 2. Suppose that the function $\xi(A)$ defined on the ring $\mathcal{R}$ is homogeneous, i.e. the distribution of $\xi(A)$ depends on the measure of the set $A$ only, but it does not depend on its position. In this case in order to have $\xi^{*}(B)<\infty$ it is necessary and sufficient that $|B|<\infty$.

Namely, if $|B|<\infty$ and $B_{1}, B_{2}, \ldots$ is a sequence of bounded disjoint Borel sets for which $B=\sum_{k=1}^{\infty} B_{k}$, then there exists a bounded Borel set $A$ and a sequence $A_{k}$ consisting of disjoint Borel sets such that

$$
A=\sum_{k=1}^{\infty} A_{k}, \quad\left|B_{k}\right|=\left|A_{k}\right| \quad(k=1,2, \ldots) .
$$

Since

$$
\sum_{k=1}^{\infty}\left|1-f\left(t, B_{k}\right)\right|=\sum_{k=1}^{\infty}\left|1-f\left(t, A_{k}\right)\right|<\infty,
$$

it follows that the sum of the series $\sum_{k=1}^{\infty} \xi^{*}\left(B_{k}\right)$ is finite with probability 1, i.e. $\xi^{*}(B)<$ $\infty$. Conversely, if $|B|=\infty$, then let $B_{1}, B_{2}, \ldots$ be disjoint Borel sets such that $B=$ $\sum_{k=1}^{\infty} B_{k},\left|B_{k}\right|=1$. In this case

$$
\sum_{k=1}^{\infty}\left|1-f\left(t, B_{k}\right)\right|=\left|1-f\left(t, B_{1}\right)\right|+\left|1-f\left(t, B_{1}\right)\right|+\cdots=\infty,
$$


therefore the series $\sum_{k=1}^{\infty} \xi^{*}\left(B_{k}\right)$ is not convergent. Thus, according to the 0 or 1 law, the equality

$$
\sum_{k=1}^{\infty} \xi^{*}\left(B_{k}\right)=\xi^{*}(B)=\infty
$$

has the probability 1 , and thus we have proved our statement.

\section{THE PROPERTIES OF COMPLETELY ADDITIVE SET FUNCTIONS DEFINED ON A $\sigma$-RING}

\section{$\S 1$. Set functions of bounded variation}

In Chapter III we have seen that if $\xi(A)$ is a completely additive set function defined on a $\sigma$-ring $\mathcal{S}$, then for every positive $T$ and $\varepsilon$ the set functions

$$
\sup _{|t| \leq T}|1-f(t, A)|, \quad \int_{|x| \leq \varepsilon} x^{2} \mathrm{~d} F(x, A), \quad \int_{|x| \leq \varepsilon} x \mathrm{~d} F(x, A), \quad \mathbf{P}(|\xi(A)|>\varepsilon)
$$

are of bounded variation. Starting from this fact, we shall prove two theorems.

THEOREM 4.1. Let $\xi(A)$ be a completely additive set function defined on a $\sigma$-ring $\mathcal{S}$ and $g(x)$ a polynomial for which $g(0)=0$. If the point 0 is not a limiting point of the closed interval $[a, b]$, then the set function

$$
\int_{a \leq x \leq b} g(x) \mathrm{d} F(x, A)
$$

is of bounded variation.

PROOF. It is clear that it suffices to prove that the set functions

$$
\int_{a \leq x \leq b} x \mathrm{~d} F(x, A), \quad \int_{a \leq x \leq b}|x|^{k} \mathrm{~d} F(x, A) \quad(k \leq 2)
$$

are of bounded variation. Let $c_{1}=\min (|a|,|b|), c_{2}=\max (|a|,|b|)$. If $a<0<b$, then

$$
\begin{aligned}
& \left|\int_{a \leq x \leq b} x \mathrm{~d} F(x, A)\right| \leq\left|\int_{|x| \leq c_{1}} x \mathrm{~d} F(x, A)\right|+c_{2} \mathbf{P}\left(|\xi(A)|>c_{1}\right), \\
& \int_{a \leq x \leq b}|x|^{k} \mathrm{~d} F(x, A) \leq \int_{|x| \leq 1} x^{2} \mathrm{~d} F(x, A)+c_{2}^{k} \mathbf{P}(|\xi(A)|>1) .
\end{aligned}
$$


On the other hand, if $b<0$ or $a>0$, then

$$
\begin{aligned}
& \left|\int_{a \leq x \leq b} x \mathrm{~d} F(x, A)\right| \leq \int_{a \leq x \leq b}|x| \mathrm{d} F(x, A) \leq c_{2} \mathbf{P}\left(|\xi(A)| \geq c_{1}\right), \\
& \int_{a \leq x \leq b}|x|^{k} \mathrm{~d} F(x, A) \leq c_{2}^{k} \mathbf{P}\left(|\xi(A)| \geq c_{1}\right),
\end{aligned}
$$

hence our statement is proved.

REMARK. In Chapter $\mathrm{V}$ we shall see that the set function $\int_{|x| \leq \varepsilon}|x| \mathrm{d} F(x, A)$ is not always of bounded variation.

TheOREM 4.2. Let $\xi(A)$ be a completely additive set function defined on a $\sigma$-ring $\mathcal{S}$ and $h(x)$ be a Borel measurable function for which

$$
|h(x)| \leq c, \quad h(x)=o\left(x^{2}\right) \quad \text { if } \quad x \rightarrow 0
$$

where $c$ is a constant. Form these conditions it follows that the set function

$$
\int_{-\infty}^{\infty} h(x) \mathrm{d} F(x, A)
$$

is of bounded variation.

Proof. By assumption there is a positive $\varepsilon$ and a constant $K$ such that $|h(x)| \leq K x^{2}$ if $|x| \leq \varepsilon$. Thus we obtain that

$$
\left|\int_{-\infty}^{\infty} h(x) \mathrm{d} F(x, A)\right| \leq \int_{-\infty}^{\infty}|h(x)| \mathrm{d} F(x, A) \leq K \int_{|x| \leq \varepsilon} x^{2} \mathrm{~d} F(x, A)+c \mathbf{P}(|\xi(A)|>\varepsilon) .
$$

Since on the right hand side there stand set functions of bounded variation, our statement is proved.

\section{$\S 2$. A further convergence theorem}

Let $\xi(A)$ be a completely additive set function defined on a $\sigma$-ring $\mathcal{S}$ and $A_{n} \in \mathcal{S}$ a convergent sequence, $\lim _{n \rightarrow \infty} A_{n}=A$. Theorem 3.2 states that the sequence of random variables $\xi\left(A_{n}\right)$ converges stochastically to $\xi(A)$. Besides, if $A_{n}$ is a monotonic sequence, the complete additiveness of $\xi$ implies the more stronger relation

$$
\lim _{n \rightarrow \infty} \xi\left(A_{n}\right)=\xi(A) .
$$

In the following theorem we shall suppose regarding the set function $\xi(A)$ only that to disjoint sets there belong independent variables. Since we only permit non-negativevalued random variables, the theorem can be proved in the same way as the corresponding theorem concerning ordinary measures. 
TheOREm 4.3. Let $\mathcal{S}$ be a $\sigma$-ring. To each element $A$ of $\mathcal{S}$ let a non-negative random variable $\xi(A)$ correspond in such a manner that if $A_{1}, A_{2}, \ldots$ is a sequence of disjoint sets of $\mathcal{S}, A=\sum_{k=1}^{\infty} A_{k}$, then

$$
\xi(A)=\sum_{k=1}^{\infty} \xi\left(A_{k}\right) .
$$

In this case the set function $\xi$ has the following property: if $B_{1}, B_{2}, \ldots$ is a convergent sequence of sets belonging to the $\sigma$-ring $\mathcal{S}, \lim _{n \rightarrow \infty} B_{n}=B$, then

$$
\lim _{n \rightarrow \infty} \xi\left(B_{n}\right)=\xi(B)
$$

Proof. Put

$$
C_{n}=B_{n} B_{n+1} \ldots, \quad D_{n}=B_{n}+B_{n+1}+\cdots
$$

Since

$$
C_{n} \subseteq B_{n} \subseteq D_{n} \quad(n=1,2, \ldots),
$$

it follows that

$$
\xi\left(C_{n}\right) \leq \xi\left(B_{n}\right) \leq \xi\left(D_{n}\right) \quad(n=1,2, \ldots) .
$$

On the other hand,

$$
\lim _{n \rightarrow \infty} C_{n}=\lim _{n \rightarrow \infty} D_{n}=\lim _{n \rightarrow \infty} B_{n}=B,
$$

consequently, by the relation (4.1) we obtain that for the monotonous sequences $C_{n}$ and $D_{n}$ of sets

$$
\lim _{n \rightarrow \infty} \xi\left(C_{n}\right)=\lim _{n \rightarrow \infty} \xi\left(D_{n}\right)=\xi(B) .
$$

From (4.2) and (4.3) it follows that

$$
\lim _{n \rightarrow \infty} \xi\left(B_{n}\right)=\xi(B)
$$

Thus we have proved the theorem.

It is an open question whether the relation

$$
\xi\left(A_{n}\right) \rightarrow \xi(A) \quad \text { if } \quad A_{n} \rightarrow A, \quad A_{n} \in \mathcal{S}
$$

holds always for a completely additive set function $\xi(A)$ defined on a $\sigma$-ring $\mathcal{S}$. In some particular cases, however, this stronger convergence holds even for set functions $\xi(A)$ which take on positive and negative values equally. The authors wishes to return to these problems in a forthcoming paper. 


\section{$\S 3$. Continuous and complete set functions}

Let $\xi$ be a $\sigma$-ring consisting of some subsets of a set $H$. Suppose that the elements of $H$ belong to $\mathcal{S}$. A completely additive set function $\xi(A)$ defined on the $\sigma$-ring $\mathcal{S}$ will be called continuous if for every element $h$ of the set $H$ we have

$$
\xi(h)=0 .
$$

The set function $\xi(A)$ will be called purely discontinuous if there exists an enumerable set $H_{1}$ such that

$$
\xi\left(A \bar{H}_{1}\right)=0 \quad \text { if } \quad A \in \mathcal{S} .
$$

If $\xi(A)$ is a completely additive set function and $\xi(h) \neq 0$ where $h \in H$, then the point $h$ will be called a discontinuity point of $\xi(A)$.

In the theory of real-valued set functions it is well known that every completely additive set function possessing points of discontinuity can be decomposed into the sum of a continuous and a purely discontinuous set function. A similar decomposition can be carried out here, too. Before passing to this we prove the following

THEOREM 4.4. Let $\xi(A)$ be a completely additive set function defined on a $\sigma$-ring $\mathcal{S}$. If $T$ is a fixed positive number, then the set function $\xi(A)$ is absolutely continuous with respect to the measure $W(T, A),{ }^{7}$ i.e.

$$
\xi(A)=0 \quad \text { if } \quad W(T, A)=0 .
$$

Proof. Since

$$
|1-f(t, A)| \leq W(T, A) \quad \text { if } \quad A \in \mathcal{S},
$$

it follows that

$$
f(t, A)=1 \quad \text { if } \quad|t| \leq T .
$$

From the inequality (1.3) it follows that, for every $t, f(t, A)=1$, what was to be proved.

Now we shall prove

TheOREm 4.5. Let $\xi(A)$ be a completely additive set function defined on a $\sigma$-ring $\mathcal{S}$. Suppose that the elements of $H$ belong to $\mathcal{S}$ also and $\xi(A)$ has at least one discontinuity point. In this case there exist continuous and purely discontinuous completely additive set functions $\xi^{\prime}(A)$ and $\xi^{\prime \prime}(A)$, resp., such that

$$
\xi(A)=\xi^{\prime}(A)+\xi^{\prime \prime}(A) \quad \text { if } \quad A \in \mathcal{S} .
$$

\footnotetext{
${ }^{7}$ See p. 244.
} 
Proof. Let $T$ be a fixed positive number. As $W(T, A)$ is a finite measure, there exists an enumerable set $H_{1}$ such that

$$
W(T, h)=0 \quad \text { if } \quad h \in H-H_{1} .
$$

Since $\xi(A)$ is absolutely continuous, regarding the measure $W(T, A)$, it follows that the set function

$$
\xi^{\prime}(A)=\xi\left(A \bar{H}_{1}\right)
$$

is continuous. On the other hand, the set function

$$
\xi^{\prime \prime}(A)=\xi\left(A H_{1}\right)
$$

is purely discontinuous and

$$
\xi(A)=\xi\left(A \bar{H}_{1}\right)+\xi\left(A H_{1}\right)
$$

Thus our theorem is proved.

In the same way as in case of ordinary set functions we can introduce also here the notion of completeness. The definition is also a perfect analogue to that on p. 34 of [6] therefore we do not consider it in detail. Taking Theorem 4.4 into account we can easily see that the process of completion can also be carried out without any difficulty.

\section{EXAMPLES}

1. Poisson set functions. Denote $M(A)$ a real-valued, finite, non-negative, additive set function defined on a ring $\mathcal{R}$. Further let $\xi(A)(A \in \mathcal{R})$ be an additive set function. If

$$
\mathbf{P}(\xi(A)=k)=\frac{M^{k}(A)}{k !} e^{-M(A)} \quad(k=0,1,2, \ldots),
$$

then the set function $\xi(A)$ will be called to be of Poisson type. In this case the characteristic function of $\xi(A)$ has the form

$$
f(t, A)=e^{M(A)\left(e^{i t}-1\right)} .
$$

If the set function $M(A)$ is completely additive (in other words: if $M(A)$ is a measure), the same holds for the set function $\xi(A)$, too. Namely, let $A_{1}, A_{2}, \ldots$ be a non-increasing sequence of sets consisting of the elements of $\mathcal{R}, \lim _{n \rightarrow \infty} A_{n}=0$, then, from the relations

$$
|1-f(t, A)| \leq M(A)|t| e^{M(A)|t|}
$$

and

$$
\lim _{n \rightarrow \infty} M\left(A_{n}\right)=0,
$$


it follows that

$$
f\left(t, A_{n}\right) \Rightarrow 1 \quad \text { if } \quad n \rightarrow \infty,
$$

hence by Theorem $2.1 \xi(A)$ is completely additive. If the measure $M(A)$ is bounded, then, by (5.1), the set function $|1-f(t, A)|$ is of bounded variation, hence $\xi(A)$ can be extended. Particularly, if $H=R_{1}, M(A)=c|A|$ where $c$ is a constant, we get the set function generated by the differences of an ordinary homogeneous Poisson process.

2. Composed Poisson set functions. Let $M_{1}(A), M_{2}(A), \ldots$ be a sequence of real-valued, non-negative, additive set functions defined on a ring $\mathcal{R}$. The additive set function $\xi(A)$ defined on the ring $\mathcal{R}$ will be called to be of composed Poisson type if the characteristic function of $\xi(A)$ has the form

$$
f(t, A)=\exp \sum_{k=1}^{\infty} M_{k}(A)\left(e^{i \lambda_{k} t}-1\right),
$$

where the set $\left\{\lambda_{k}\right\}$ is the set of all possible values of the random variables $\xi(A)(A \in \mathcal{R})$ and

$$
\sum_{k=1}^{\infty} M_{k}(A)<\infty \quad(A \in \mathcal{R}) .
$$

If

$$
M(A)=\sum_{k=1}^{\infty} M_{k}(A) \quad(A \in \mathcal{R})
$$

is a finite measure on the ring $\mathcal{R}$, then in the same way as in the case of Poisson set functions it can be shown that $\xi(A)$ is completely additive. If, in addition, we suppose that

$$
\sum_{k=1}^{\infty}\left|\lambda_{k}\right| M_{k}(A)<\infty \quad(A \in \mathcal{R})
$$

and the sum (5.3) is a bounded measure on the $\operatorname{ring} \mathcal{R}$, then by the relation (5.2) we obtain that

$$
\begin{aligned}
|1-f(t, A)| & =\left|\prod_{k=1}^{\infty} e^{M_{k}(A)\left(e^{i \lambda_{k} t}-1\right)}-1\right| \leq \sum_{k=1}^{\infty}\left|e^{M_{k}(A)\left(e^{i \lambda_{k} t}-1\right)}-1\right| \\
& \leq|t| \sum_{k=1}^{\infty}\left|\lambda_{k}\right| M_{k}(A) e^{\lambda_{k} t M_{k}(A)} \leq L(t) \sum_{k=1}^{\infty}\left|\lambda_{k}\right| M_{k}(A)
\end{aligned}
$$

where

$$
L(t)=\max _{k} e^{\left|\lambda_{k} t\right| M_{k}(A)},
$$


hence $|1-f(t, A)|$ is of bounded variation for every fixed value of $t$; thus $\xi(A)$ can be extended to the $\sigma$-ring $\mathcal{S}(\mathcal{R})$.

It is easy to see that if $\mathcal{R}$ is a ring of some subsets of the space $\mathcal{R}_{n}$ and the distribution of $\xi(A)$ depends on the measure $|A|$ of the set $A$ only, then

$$
M_{k}(A)=c_{k}|A| \quad(k=1,2, \ldots)
$$

where $c_{1}, c_{2}, \ldots$ are constants.

3. Laplace-Gauss set function. That is the name of additive set functions $\xi(A)$ for which

$$
f(t, A)=e^{i t M(A)-D^{2}(A) \frac{t^{2}}{2}} \quad(A \in \mathcal{R}),
$$

where $M(A)$ and $D^{2}(A)$ are real-valued, additive set functions, $D^{2}(A) \geq 0(0 \in \mathcal{R})$. If both set functions $M(A)$ and $D^{2}(A)$ are completely additive, then taking into account Theorem 2.1 it follows that $\xi(A)$ is completely additive, too. If, in addition, the set functions $M(A)$ and $D^{2}(A)$ are bounded, then from the inequalities

$$
|1-f(t, A)| \leq\left|1-e^{i t M(A)}\right|+1-e^{-D^{2}(A) \frac{t^{2}}{2}} \leq|t| M(A)+D^{2}(A) \frac{t^{2}}{2}
$$

it follows that for any fixed value of $t$ the set function $|1-f(t, A)|$ is of bounded variation, therefore $\xi(A)$ can be extended to $\mathcal{S}(\mathcal{R})$. If $\mathcal{R}$ is a ring of certain subsets of the space $R_{n}$ and the distribution of $\xi(A)$ depends on $|A|$ only, then it is easy to see that

$$
M(A)=M|A|, \quad D^{2}(A)=D^{2}|A|,
$$

where $M$ and $D^{2}$ are constants. In particular, if $n=1$, then $\xi(A)$ is nothing else than the set function generated by the differences of the ordinary Brownian movement process.

4. Let $f_{n}(x)$ denote the $n$-th Rademacher function

$$
f_{n}(x)=\operatorname{sg} \sin 2^{n} \pi x \quad(0 \leq x \leq 1)
$$

Let the interval $[0,1]$ be the space of the elementary events and the possible events be the Lebesgue measurable sets of this interval. Then the functions $\left\{f_{n}(x)\right\}$ will be independent random variables. Consider the random variables

$$
g_{n}(x)=\frac{f_{n}(x)}{n} \quad(n=1,2, \ldots) .
$$

Since

$$
M\left(g_{n}(x)\right)=0, \quad D^{2}\left(g_{n}(x)\right)=\frac{1}{n^{2}}
$$

it follows that the series

$$
\sum_{n=1}^{\infty} g_{n}(x)
$$


converges with probability 1 regardless of the order of summation. If $A$ is a set of natural numbers, then the series

$$
\sum_{n \in A} g_{n}(x)
$$

converges with probability 1 in every rearrangement to the same function, further the set function

$$
g(x, A)=\sum_{n \in A} g_{n}(x)
$$

is completely additive on the $\sigma$-ring $\mathcal{S}$ of the subsets of the set $H$ of natural numbers ([4], p. 118, Corollary 1). The set function $g(x, A)$ has the following properties:

a) $\mathbf{P}\left(\left|g_{n}(x)\right|=\frac{1}{n}\right)=1$, and thus the series

$$
\sum_{n=1}^{\infty}\left|g_{n}(x)\right|
$$

diverges. Hence it follows that the set function $g(x, A)$ can not be decomposed in such a way that

$$
g(x, A)=g^{+}(x, A)-g^{-}(x, A),
$$

where $g^{+}(x, A), g^{-}(x, A)$ are completely additive, non-negative set functions. Namely, if such a decomposition would exist, then from the inequality

$$
\sum_{n=1}^{\infty}\left|g_{n}(x)\right| \leq g^{+}(x, H)+g^{-}(x, H)
$$

it would follow that the series $\sum_{n=1}^{\infty} g_{n}(x)$ converges absolutely, what is not true.

b) Since for every positive $\varepsilon$ we have

$$
M\left(\left|g_{n}(x)\right|\right)=\int_{|x| \leq \varepsilon}|x| \mathrm{d} F(x, n) \quad \text { if } \quad n \geq \frac{1}{\varepsilon},
$$

then, by Condition a), it follows that the set function

$$
\int_{|x| \leq \varepsilon}|x| \mathrm{d} F(x, A)
$$

is not of bounded variation. 


\section{References}

[1] A. Blanc-Lapierre et R. Fortet, Sur les répartitions de Poisson, Comptes Rendus Acad. Sci. Paris, 240 (1955), pp. 1045-1046.

[2] S. Bochner, Stochastic processes, Annals of Math., 48 (1947), pp. 1041-1061.

[3] H. Cramér, A contribution to the theory of stochastic processes, Proc. Sec. Berkeley Symp., (1951), pp. 329-340.

[4] J. L. Doob, Stochastic processes (New York-London, 1953).

[5] B. V. Gnedenko i A. N. Kolmogorov, Prevedel'nye raspredeleniâ dlâ summ nezavisimyh slučajnyh veličin (Moszkva-Leningrad, 1949).

[6] H. Hahn and A. Rosenthal, Set functions (New Mexico, 1948).

[7] E. Hopf, Ergodentheorie (Berlin, 1937).

[8] A. N. Kolmogorov, Grundbegriffe der Wahrscheinlichkeitsrechnung (Berlin, 1933).

[9] E. Marczewski, Remarks on the Poisson stochastic process. II, Studia Mathematica, 13 (1953), pp. 130-136.

[10] A. PrÉKopa, On the convergence of series of independent random variables, Publ. Math. Debrecen, 4 (1956), pp. 410-417.

[11] A. PrÉKOPA, Extension of multiplicative set functions with values in a Banach algebra, Acta Math. Acad. Sci. Hung., 7 (1956), pp. 201-213.

[12] C. Ryll-Nardzewski, On the non-homogeneous Poisson process. I, Studia Mathematica, 14 (1954), pp. 124-128. 